\title{
On the scalability of inexact balancing domain decomposition by constraints with overlapped coarse/fine corrections
}

\author{
Santiago Badia ${ }^{\mathrm{a}, \mathrm{b}}$, Alberto F. Martín ${ }^{\mathrm{a}, \mathrm{b}}$, Javier Principe ${ }^{\mathrm{a}, \mathrm{b}}$ \\ ${ }^{a}$ Centre Internacional de Mètodes Numèrics en Enginyeria (CIMNE), Parc Mediterrani de \\ la Tecnologia, UPC, Esteve Terradas 5, 08860 Castelldefels, Spain \\ (\{sbadia, amartin, principe\}@cimne.upc .edu) \\ ${ }^{b}$ Universitat Politècnica de Catalunya, Jordi Girona 1-3, Edifici C1, 08034 Barcelona, \\ Spain
}

\begin{abstract}
In this work, we analyze the scalability of inexact two-level Balancing Domain Decomposition by Constraints (BDDC) preconditioners for Krylov subspace iterative solvers, when using a highly scalable asynchronous parallel implementation where fine and coarse correction computations are overlapped in time. This way, the coarse-grid problem can be masked by fine-grid computations (which are embarrassingly parallel) in a wide range of cases. Further, we consider inexact solvers to reduce the computational cost/complexity and memory consumption of coarse and local problems and boost the scalability of the solver. Out of our numerical experimentation, we conclude that the BDDC preconditioner is quite insensitive to inexact solvers. In particular, one cycle of algebraic multigrid (AMG) is enough to attain algorithmic scalability. Further, the clear reduction of computing time and memory requirements of inexact solvers compared to sparse direct ones makes possible to scale far beyond state-of-the-art BDDC implementations. Excellent weak scalability results have been obtained with the proposed inexact/overlapped implementation of the two-level BDDC preconditioner, up to 93,312 cores and 20 billion unknowns on JUQUEEN.
\end{abstract}

Keywords: Domain decomposition, inexact solvers, coarse-grid correction, BDDC, parallelization, overlapping, MPMD, scalability

\section{Introduction}

In order to deal with increasing levels of complexity in the simulation of phenomena governed by partial differential equations (PDEs), computational engineering and science must advance in the development of numerical algorithms and implementations that will efficiently exploit the ever-increasing amount of computational resources. The growth in computational power that resulted from Moore's law passes now through increasing the number of cores in a chip, 
instead of making cores faster. As a result, the next generation of supercomputers, able to reach 1 exaflop/s, is expected to reach billions of cores. The efficient exploitation of billion-fold levels of concurrency is a big challenge. The advance of large scale scientific computing will be strongly related to the ability to efficiently exploit these extreme core counts [1].

The time spent in an implicit simulation at the linear solver relative to the overall execution time grows with the size of the problem and the number of cores [2]. For extreme scale implicit simulations, a massively parallel linear solver is a key component. The efficient exploitation of billion-fold levels of concurrency at the linear system solve is a big challenge. This scenario exacerbates the need of highly scalable algorithms and implementations. Only numerical algorithms with all their components scalable will efficiently run on extreme scale supercomputers. Extreme scale solvers should be developed under the assumption that local flops are cheap and communications expensive. On extreme core counts, it will be a must to reduce communication and synchronization among processors, and overlap communication with computation. At the largest scales, linear solvers are based on preconditioned Krylov subspace methods. Algorithmically scalable preconditioners include (algebraic) multigrid (MG) [3] and some

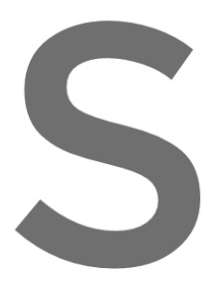

domain decomposit
is not enough for pr
allow for a massivel
s/implementations
coarse problems to
loss of sparsity and

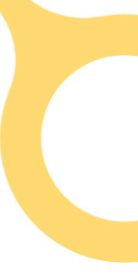

DD prect

dition
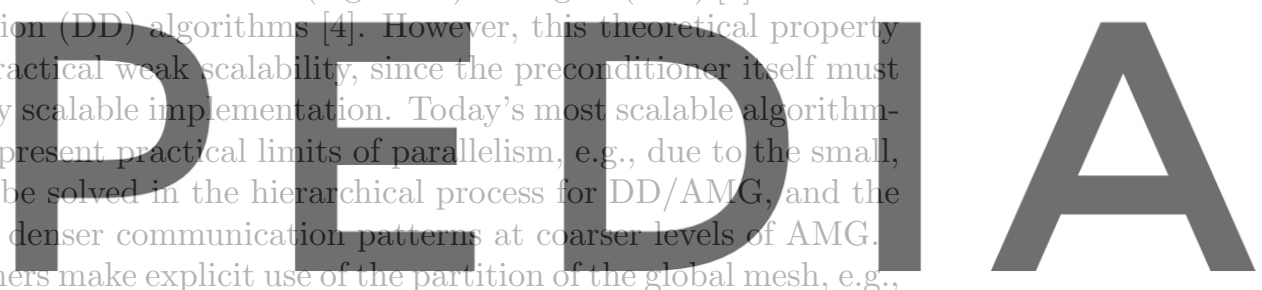

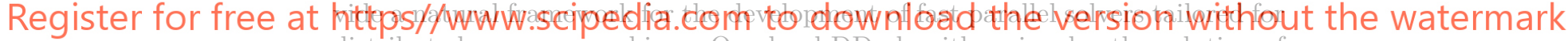

distributed-memory machines. One-level DD algorithms involve the solution of

local problems and nearest-neighbors communications. A (second level) coarse correction (coupling all subdomains) is required to have algorithmic scalability, but it also harms the practical (CPU time) weak scalability. Two-level DD algorithms include the Balancing Neumann-Neumann preconditioner (BNN) [5], the Balancing DD by Constraints preconditioner (BDDC) [6], and FETI-DP preconditioners [7]. In all these cases, for positive-definite matrices, a polylogarithmic expression of the condition number of the preconditioned system $\kappa=1+\log ^{2}\left(\frac{H}{h}\right)$ can be proved, where $h$ and $H$ are the mesh and subdomain characteristic sizes, respectively, and $d$ is the space dimension; $\left(\frac{H}{h}\right)^{d}$ is the local problem size. Consequently, in weak scaling scenarios, i.e., increasing the linear system size and number of processors keeping $\frac{H}{h}$ fixed, the number of iterations of the preconditioned conjugate gradient (PCG) solver is (asymptotically) independent of the number of processors.

The practical scalability limits of a two-level DD implementation is determined by the coarse solver computation, whose size increases (at best) linearly with respect to the number of subdomains. The coarse problem rapidly becomes the bottleneck of the algorithm as we increase the number of processors, 
reducing weak scalability [8]. The coarse problem is several orders of magnitude smaller than the original global system, and only a very small portion of the computing cores can efficiently be exploited (assuming a parallel coarse solver). In typical DD implementations, it produces an unacceptable parallel efficiency loss, since all the cores not involved in the coarse solver computation are idling (see Figure 1). One obvious strategy to improve scalability is to reduce the wall-clock time spent at the coarse solver by using, e.g., a MPI-distributed sparse direct solver like MUMPS [9] (see [10] for BDDC and [11] for FETI-DP). However, this approach only mitigates the problem.

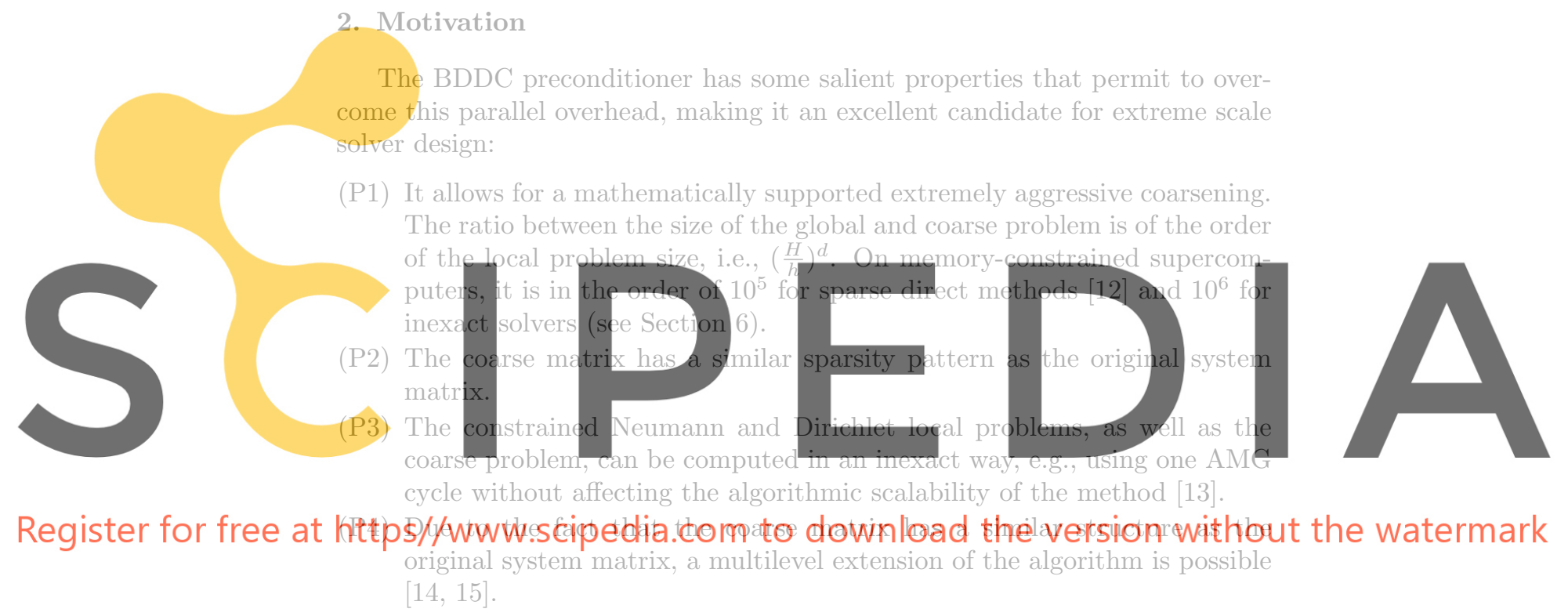

(P5) Coarse and fine components can be computed in parallel, since the basis for the coarse space is constructed in such a way that it is orthogonal to the fine component space with respect to the inner product endowed by the system matrix [12].

Properties (P1) and (P2) are readily exploited in any BDDC implementation. Property (P3), i.e., the algorithmic scalability of BDDC with inexact solvers, has been proved by Dohrmann in [13]. Similar inexact preconditioners have been presented in [16]. The inexact BDDC method can easily increase parallel efficiency, due to the linear complexity of the coarse solver, especially at large core counts. However, as far as we know, a practical weak scalability analysis of inexact BDDC methods (at large scales) has not been carried out so far. Besides, for FETI-DP, one cycle of the MPI-distributed AMG solver in BoomerAMG [17] has been used as inexact coarse solver in $[18,19]$ for $2 \mathrm{D}$ elasticity problems.

With regard to (P4), a multilevel BDDC algorithm has been proposed in [15], where the coarse problem at the next BDDC level is approximated by its BDDC approximation. This way, the CPU cost of the coarse problem is reduced, but 
the condition number bound increases with the number of levels [15]. A highperformance implementation of the multilevel BDDC method can be found in [10].

The efficient exploitation of (P5), i.e., the orthogonality between coarse and fine spaces, is not trivial. However, this property makes possible a parallel computation of coarse and fine corrections, i.e., overlapped in time. In [12], we have classified all the duties in an exact (i.e., using sparse direct solvers) BDDC-PCG algorithm into fine and coarse duties. These duties have been re-scheduled to achieve the maximum degree of overlapping while preserving data dependencies. The actual implementation of this idea requires significant code refactoring, since it involves a switch from SPMD (Single Program Multiple Data) to a MPMD (Multiple Program Multiple Data) parallel execution mode; processors are divided into those having fine grid duties and those having coarse grid duties. Clearly, this approach reduces synchronization among processors, and overlaps communications/computations, following the exascale solver paradigm [1]. It has been exploited in [12], where we have performed scalability analyses for the 3D Poisson and linear elasticity problems on a pair of state-of-the-art multicore-based distributed-memory machines (HELIOS and

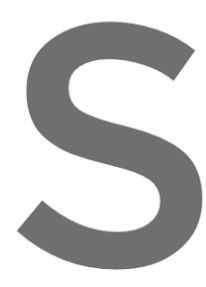

CURIE).

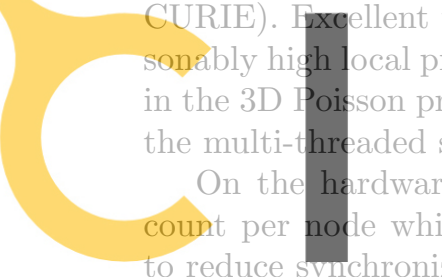

to reduce symchronization, as in the or

will be crucial at extreme core counts. Further, this overlapped implementa-

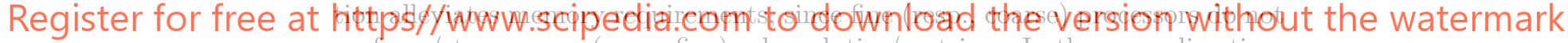
perform/store coarse (resp., fine) solver duties/matrices. In the same direction, linear complexity inexact solvers, much less memory intensive than sparse direct methods, will certainly be favored. They should also be favored at large core counts, since the potential loss of scalability due to the coarse solver is much less dramatic. The current state-of-the-art in DD implementations and the supercomputing trends to reach the exascale have motivated the combined overlapped/inexact BDDC implementation proposed in this work.

In this article, we extend the overlapped implementation in [12] for exact solvers to the inexact BDDC methods proposed in [13] (with slight modifications). First, we analyze the effect of perturbing in isolation every problem at the BDDC preconditioner. Next, we propose different inexact methods, combining different numbers of AMG cycles for each internal problem. A comprehensive weak scalability analysis of the resulting overlapped/inexact BDDC implementation has been performed till 93,312 cores and more than 20 billion unknowns on JUQUEEN, at the Jülich Supercomputing Center (JSC). As far as we know, these are the largest scale scalability analyses and simulations performed so far with DD methods.

This work is structured as follows. Section 3 is devoted to non-overlapping $\mathrm{DD}$ and the BDDC preconditioner whereas Section 4 is devoted to the intro- 
duction of inexact variants. In Section 5, we extend the highly scalable parallel distributed-memory implementation of the BDDC algorithm in [12], which overlaps fine and coarse computations, to the inexact variant. In Section 6, we report a comprehensive set of numerical experiments that includes a study of the influence of approximately solving each internal problem in isolation and a weak scalability analysis of strategically selected combinations. Finally, in Section 7, we draw some conclusions and define future lines of work.

\section{Balancing Domain Decomposition}

\subsection{Problem setting}
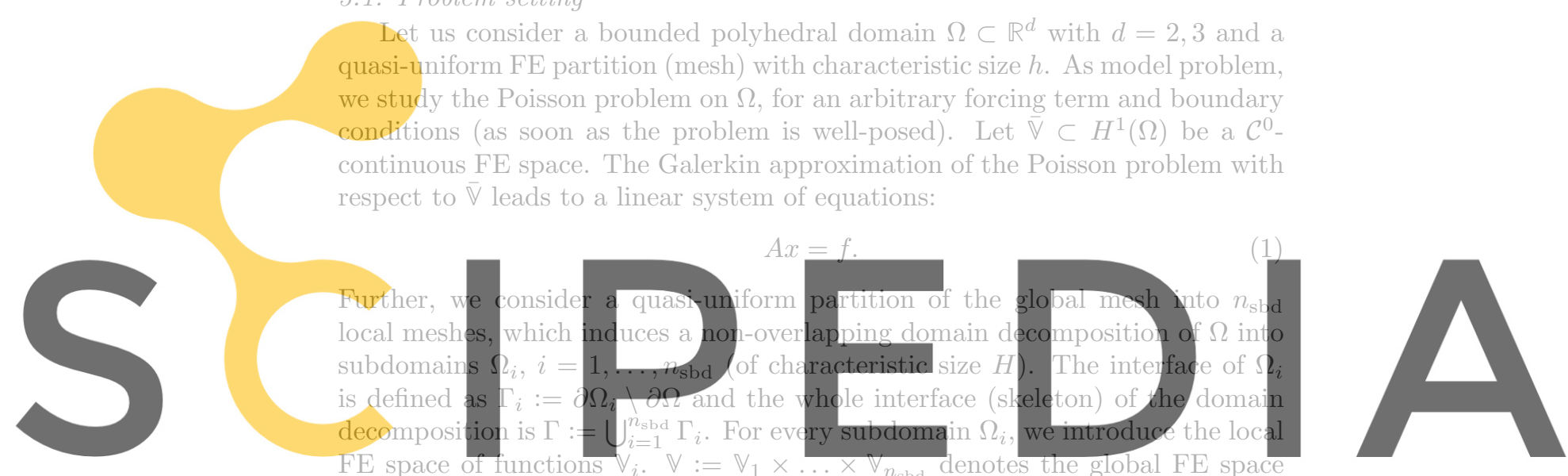

Register for free at https //wismw schipedialcomprtodownload the version without the watermark

of functions that can be discontinuous on $\Gamma$; clearly, $\overline{\mathbb{V}} \subset \mathbb{V}$. Obviously, all FH

Let us define the restriction operator $R_{i}: \bar{V} \rightarrow \mathbb{V}_{i}$, that applied to a vector in

$\bar{\nabla}$ provides its restriction into $\Omega_{i}$, and $R:=R_{1} \times \ldots \times R_{n_{\text {sbd }}}: \bar{\nabla} \rightarrow \mathbb{V}$. Let us also

define the projector $E_{i}:=R_{i}^{t} D_{i}: \mathbb{V}_{i} \rightarrow \overline{\mathbb{V}}$, where $D_{i}: \mathbb{V}_{i} \rightarrow \mathbb{V}_{i}$ is a weighting operator. The weighting operators represent a partition of unity, in the sense that $R^{t} D R=I$, with $D:=D_{1} \times \ldots \times D_{n_{\text {sbd }}}: \mathbb{V} \rightarrow \mathbb{V}$. Further, let $E:=R^{t} D$.

The subdomain FE matrix corresponding to $\mathbb{V}_{i}$ is denoted by $K^{(i)}$, and its size is denoted by $n_{i} . K:=\operatorname{diag}\left(K^{(1)}, \ldots, K^{\left(n_{\mathrm{sbd}}\right)}\right)$ is the global sub-assembled FE matrix on $\mathbb{V}$. (Along the paper, we denote with the letter $K$ (partially) subassembled matrices and with $A$ fully assembled ones.) Analogously, we define the local sub-assembled right-hand side $g^{(i)}$ and its global counterpart $g$. The system matrix $A$ and right-hand side $f$ can be obtained after the assembly of $K$ as $A=R^{t} K R$ and $g$ as $f=R^{t} g$.

The non-overlapping partition induces a reordering of FE vectors into interior and interface nodes, i.e., $u=\left[u_{I}, u_{\Gamma}\right]^{t}$. We also define the interior restriction operator $R_{I} u:=u_{I}$. It leads to the following block reordered structure of the global assembled, global sub-assembled and local matrices:

$$
A=\left[\begin{array}{cc}
A_{I I} & A_{I \Gamma} \\
A_{\Gamma I} & A_{\Gamma \Gamma}
\end{array}\right], \quad K=\left[\begin{array}{cc}
A_{I I} & K_{I \Gamma} \\
K_{\Gamma I} & K_{\Gamma \Gamma}
\end{array}\right], \quad \text { and } \quad K^{(i)}=\left[\begin{array}{cc}
A_{I I}^{(i)} & A_{I \Gamma}^{(i)} \\
A_{\Gamma I}^{(i)} & K_{\Gamma \Gamma}^{(i)}
\end{array}\right],
$$


respectively. Matrices $A_{I I}, A_{I \Gamma}, A_{\Gamma I}$ and $K_{\Gamma \Gamma}$ present a block diagonal structure (very amenable to parallelization), e.g., $A_{I I}=\operatorname{diag}\left(A_{I I}^{(1)}, A_{I I}^{(2)}, \ldots, A_{I I}^{\left(n_{\text {sbd }}\right)}\right)$. Matrices $K_{I \Gamma}$ and $K_{\Gamma I}$ are simply an extension by zeros of $A_{I \Gamma}$ and $A_{\Gamma I}$, respectively.

\section{2. $B D D C$ preconditioner}

The BDDC preconditioner is a two-level domain decomposition method where some local fine-grid corrections and a global coarse-grid correction (that couples all subdomains and makes the preconditioner both scalable and optimal)

are combined. The idea behind the BDDC preconditioner is to approximate the original FE problem by another one in which we relax the continuity conditions, drastically reducing the size of the modified Schur complement, combined with an initial and final interior correction.

The construction of the BDDC preconditioner requires a partition of the degrees of freedom (DoFs) on $\Gamma$ into objects, which can be corners, edges or faces. Next, we associate to some (or all) of these objects a coarse DoF. The coarse DoFs can be the values of the function at the corners, or the mean

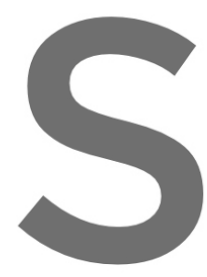
values of the the subsp $\bar{\nabla} \subset \dot{V} \subset \mathbb{V}$ as BDDC corner coa coarse DoH
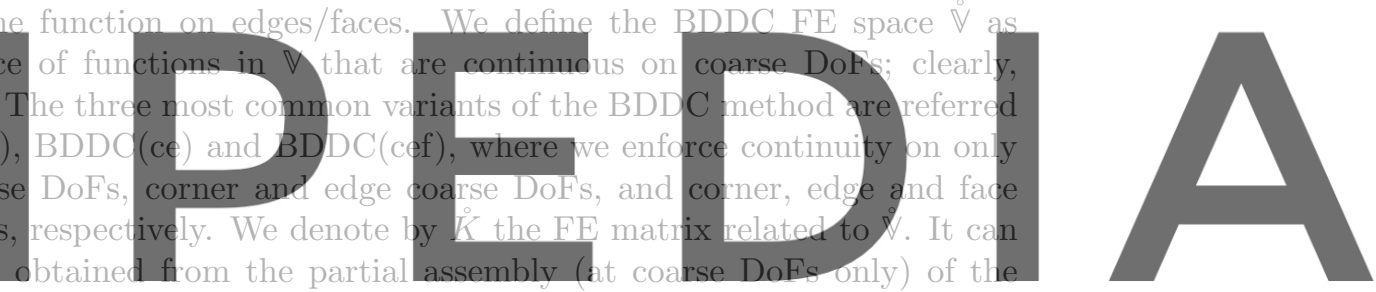

global sub-assembled matrix $K$, even though it is never implemented this way.

$P_{I}:=R_{I}^{t} A_{I I}^{-1} R_{I}, \quad P_{F C}:=E K^{-1} E^{t}, \quad H:=I-P_{I} A=\left[\begin{array}{cc}0 & -A_{I I}^{-1} A_{I \Gamma} \\ 0 & I_{\Gamma}\end{array}\right]$.

( $F C$ denotes fine/coarse correction and $H$ is the so-called discrete harmonic extension operator.) The BDDC preconditioner $M$ consists in a multiplicative combination of $P_{I}, P_{F C}$, and $P_{I}$. Using the fact that $P_{I} A P_{I}=P_{I}$, we obtain:

$$
M=P_{I}+H P_{F C} H^{t} .
$$

The practical implementation of the BDDC correction $P_{F C}$ requires some elaboration. Let us consider a decomposition of the BDDC space $\mathscr{V}^{\circ}$ into a fine space $\stackrel{\vee}{V}_{F}$ of vectors that vanish on coarse DoFs and the $\stackrel{\circ}{K}$-orthogonal complement $\grave{\vee}_{C}$, denoted as the coarse space. As a result, the BDDC FE problem can be decomposed into fine and coarse components, i.e., $\stackrel{\circ}{x}=\stackrel{\circ}{K}^{-1} E^{t} r=x_{F}+x_{C}$. Since fine and coarse spaces are $\stackrel{\circ}{K}$-orthogonal by definition, they can be computed in parallel.

The fine space functions in $\stackrel{\circ}{F}_{F}$ vanish on coarse DoFs (which are the only DoFs that involve continuity among subdomains). Due to the $\stackrel{\circ}{K}$-orthogonality, 
the fine component can be defined as $x_{F}:=E_{F} K_{F}^{-1} E_{F}^{t}$, where $K_{F}$ is the Galerkin projection of $K$ onto $\stackrel{\circ}{F}_{F}$, i.e., functions that vanish on coarse DoFs, and $E_{F}$ is the restriction of $E$ to $\mathscr{V}_{F}$. In order to compute this fine correction in practice, we define the local matrix of constraints $C_{i}$ such that, given a local vector of unknowns, it provides its local coarse DoFs values. We refer to [21] for a detailed implementation of $C_{i}$. As a result, the fine correction $x_{F}$ computation only involves constrained Neumann problems:

$$
\left[\begin{array}{cc}
K^{(i)} & C_{i}^{t} \\
C_{i} & 0
\end{array}\right]\left[\begin{array}{c}
x_{F}^{(i)} \\
\lambda
\end{array}\right]=\left[\begin{array}{c}
E_{i}^{t} r \\
0
\end{array}\right]
$$

As it is described in detail in [21], the solution of the constrained Neumann problem is performed after applying a permutation that separates coarse corner DoFs (denoted by $c$ ) from the rest of DoFs (denoted by $r$ ), i.e., $x^{(i)}=\left[x_{c}^{(i)}, x_{r}^{(i)}\right]^{t} \in \mathbb{V}_{i}$. Further, we define the restriction $R_{r, i}$ such that $R_{r, i} x^{(i)}=x_{r}^{(i)}$. Corner DoFs can be explicitly eliminated (in fact $x_{c}^{(i)}=0$ for the fine correction), leading to the system
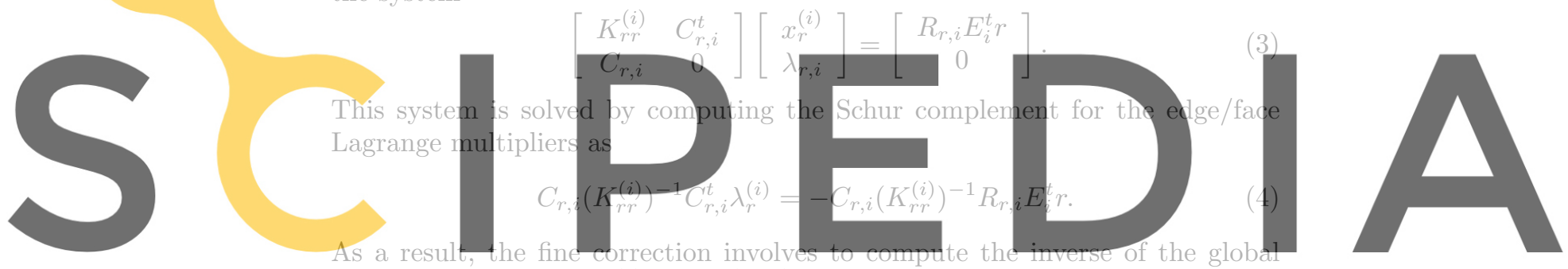

matrix $K_{r r}:=\operatorname{diag}\left(K_{r r}^{(1)} \ldots, K_{r r}^{\left(n_{\mathrm{sbd}}\right)}\right)$. There are existing mechanisms that

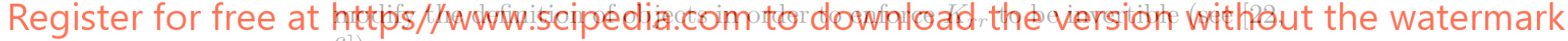
6]).

The coarse space $\mathbb{V}_{C} \subset \mathbb{V}^{\circ}$ is built as

$$
\stackrel{\circ}{\vee}_{C}=\operatorname{span}\left\{\Phi_{1}, \Phi_{2}, \ldots, \Phi_{n_{\mathrm{cts}}}\right\}
$$

where every coarse function is associated to a coarse DoF. We denote by $\Phi$ the matrix with columns $\Phi_{i}$. The coarse basis $\Phi$ (the matrix with columns $\Phi_{i}$ ) is the solution of a multiple right-hand side global system. Since the values on the coarse DoFs are prescribed and the rest of DoFs are local, the coarse space can also be computed via (parallel) local constrained Neumann problems, i.e.,

$$
\left[\begin{array}{cc}
K^{(i)} & C_{i}^{t} \\
C_{i} & 0
\end{array}\right]\left[\begin{array}{l}
\Phi^{(i)} \\
\Lambda^{(i)}
\end{array}\right]=\left[\begin{array}{l}
0 \\
I
\end{array}\right]
$$

System (5) is solved in the same way as system (2), getting:

$$
\begin{aligned}
& \Phi^{(i)}=\left[\begin{array}{l}
\Phi_{c}^{(i)} \\
\Phi_{r}^{(i)}
\end{array}\right], \quad \Phi_{c}^{(i)}=\left[\begin{array}{ll}
I & 0
\end{array}\right], \quad \Phi_{r}^{(i)}=-\left(K_{r r}^{(i)}\right)^{-1} C_{r, i}^{t} \Lambda_{r}^{i}-\left[\begin{array}{ll}
K_{r c}^{(i)} & 0
\end{array}\right], \\
& C_{r, i}\left(K_{r r}^{(i)}\right)^{-1} C_{r, i}^{t} \Lambda_{r}^{(i)}=-C_{r, i}\left(K_{r r}^{(i)}\right)^{-1}\left[\begin{array}{ll}
K_{r c}^{(i)} & I
\end{array}\right] .
\end{aligned}
$$


Let us note that any function $\Phi_{i}$ is associated to an object and its support is the set of subdomains that share this object. Thus, at every subdomain we only compute the non-zero restrictions, i.e., the coarse space basis functions related to local coarse DoFs. We compute the coarse matrix $K_{C}$ as

$$
K_{C}=\Phi^{t} K \Phi=\sum_{i=1}^{n_{\text {sbd }}} R_{C, i}^{t} \Phi^{(i)^{t}} K^{(i)} \Phi^{(i)} R_{C, i}
$$

where $R_{C, i}$ is the coarse matrix assembly operator, i.e., the local-to-global correspondence for coarse DoFs. The subdomain contributions $\Phi^{(i)^{t}} K^{(i)} \Phi^{(i)}$ can readily be computed (in parallel) and assembled, e.g., in one processor. The coarse residual $r_{C}=\Phi^{t} E^{t} r$ is computed analogously (see [21]). Once $K_{C}$ and $r_{C}$ are assembled, the coarse correction is obtained as $x_{C}=\Phi K_{C}^{-1} r_{C}$. The BDDC preconditioner can finally be stated as:

$$
M=P_{I}+H\left(E^{t} \Phi K_{C}^{-1} \Phi^{t} E+E_{F}^{t} K_{F}^{-1} E_{F}\right) H^{t} .
$$
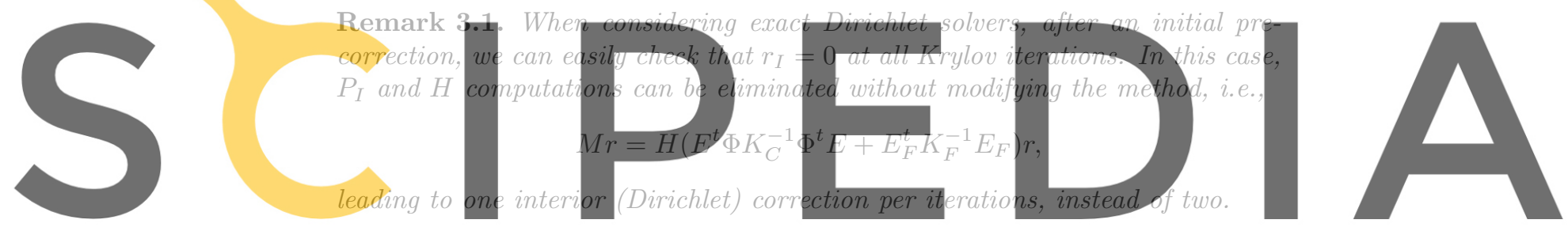

We refer to [23] for a proof of the following theorem, about the condition

Theorem 3.1. The maximum and minimum eigenvalues of the BDDC preconditioned system matrix are:

$$
\lambda_{\min }(M A) \geq 1, \quad \lambda_{\max }(M A) \leq \beta \omega, \quad \text { with } \omega:=\left(1+\log ^{2}\left(\frac{H}{h}\right)\right),
$$

for $B D D C(c)$ or $B D D C(c e)$ in $2 D$, and $B D D C(c e)$ and $B D D C(c e f)$ in $3 D$, where $\beta>0$ does not depend on $(H, h)$. For the BDDC(c) preconditioner in $3 D$,

$\lambda_{\min }(M A) \geq 1, \quad \lambda_{\max }(M A) \leq \beta^{\prime} \frac{H}{h} \omega, \quad$ for $\beta^{\prime}>0$ also independent of $(H, h)$.

Remark 3.2. The BDDC preconditioner is quasi-optimal and algorithmically scalable, since the condition number of the preconditioned system matrix only depends on the local system size, which is fixed in a weak scaling scenario. Further, the condition number is a poly-logarithmic function of $\frac{H}{h}$, with the only exception of $B D D C(c)$ in $3 D$. In this last case, the condition number is affected by an additional $\frac{H}{h}$ factor, which can be large (e.g., 60 in the numerical experiments of Section 6). It justifies the large iteration counts of this method (compared to those of $B D D C(c e)$ and $B D D C(c e f)$ ). 


\section{Inexact BDDC}

The exact BDDC preconditioner involves some linear systems to be solved. The action of $K_{r r}^{-1}$, i.e., the local constrained (on the coarse corner DoFs only) Neumann problems, is required to compute the coarse basis $\Phi$ and the fine correction, the action of $A_{I I}^{-1}$, i.e., the local Dirichlet problems, is required for the interior corrections, and the action of $K_{C}^{-1}$, i.e., the coarse problem, must be solved to compute the coarse correction. These problems are traditionally solved via sparse direct methods [6]. However, as motivated in the introduction, the use of inexact solvers is very appealing for large-scale simulations on supercomputers, due to increasing memory restrictions and higher core counts. Dohrmann has proposed and analyzed in [13] an inexact version of the BDDC method, where the local/coarse problems are replaced by preconditioners. Let us assume that we have at our disposal an approximation $\widetilde{K}_{r r}$ of $K_{r r}$ such that:

$$
\delta_{F} x^{t} K_{r r} x \leq x^{t} \widetilde{K}_{r r} x \leq \Delta_{F} x^{t} K_{r r} x, \quad \forall x .
$$

(Along this section, we assume $\delta_{(\cdot)}$ and $\Delta_{(\cdot)}$ to be positive constants independent of $(H, h)$ and the vector space for $x$ can be inferred from the matrices in the
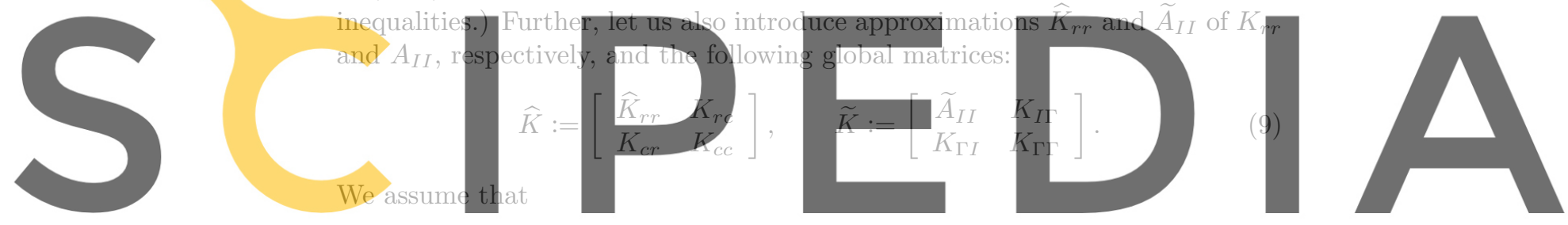

$$
\delta_{\Phi} x^{t} K x \leq x^{t} \hat{K} x \leq \Delta_{\Phi} x^{t} K x,
$$

to be semi-positive definite, we also assume

$$
x^{t} K_{r r} x \leq x^{t} \widehat{K}_{r r} x, \quad x^{t} A_{I I} x \leq x^{t} \widetilde{A}_{I I} x, \quad \forall x .
$$

As noted in [13], since $K$ is singular, the kernel of $K, \widehat{K}$, and $\widetilde{K}$ must be identical to satisfy (10). Let $W$ be such that $\operatorname{ker}(K) \subseteq \operatorname{range}(W)$, and $W_{I}=R_{I} W$. Given an arbitrary approximation $\bar{A}_{I I}$, we can build $\widetilde{A}_{I I}$ by solving exactly on range $\left(W_{I}\right)$ (see [13]), i.e.,

$\widetilde{A}_{I I}^{-1}:=W_{I}\left(W_{I}^{t} \bar{A}_{I I}^{-1} W_{I}\right)^{-1} W_{I}^{t}+E_{I} \bar{A}_{I I}^{-1} E_{I}^{t}, \quad E_{I}:=I-A_{I I} W_{I}\left(W_{I}^{t} \bar{A}_{I I}^{-1} W_{I}\right)^{-1} W_{I}^{t}$.

Due to the block-diagonal nature of $K$, this correction is local. The definition of the kernel-correction for $\widehat{K}_{r r}$ is defined analogously. Finally, for the approximation of the coarse matrix $K_{C}$ we study two different options. A difference with respect to the previous problems is the fact that $K_{C}$ is not available when using inexact solvers. (It involves the exact computation of $\Phi$.) One option is to assemble the Galerkin projection of $K$ onto the inexact coarse basis $\widehat{\Phi}$, i.e., $\widehat{\Phi}^{t} K \widehat{\Phi}$, and consider an approximation of this matrix such that:

$$
\delta_{C} x^{t}\left(\widehat{\Phi}^{t} K \widehat{\Phi}\right) x \leq x^{t} \widetilde{K}_{C} x \leq \Delta_{C} x^{t}\left(\widehat{\Phi}^{t} K \widehat{\Phi}\right) x, \quad \forall x .
$$


Another approach, the one used in [13], is to consider an approximation of the coarse matrix $\widehat{\Phi}^{t} \widehat{K} \widehat{\Phi}$ :

$$
\delta_{C} x^{t}\left(\widehat{\Phi}^{t} \widehat{K} \widehat{\Phi}\right) x \leq x^{t} \widetilde{K}_{C} x \leq \Delta_{C} x^{t}\left(\widehat{\Phi}^{t} \widehat{K} \widehat{\Phi}\right) x, \quad \forall x .
$$

Remark 4.1. In general, the inexact matrices $\widetilde{K}_{r r}, \bar{K}_{r r}$, and $\bar{A}_{I I}$ are not explicitly built, and only the action of their inverses is approximated in the algorithm, e.g., using one/several $A M G$ cycles. Further, $\widehat{K}_{r r}$, and $\widetilde{A}_{I I}$ are dense matrices, due to the kernel correction. However, when using the coarse problem approximation in (12), $\widehat{K}_{r r}$ seems to be explicitly needed. Fortunately, using the inexact version of (5) (Equations (15)-(16) below) we can easily check that $K_{r r}^{(i)} \Phi^{(i)}=-C^{t} \Lambda$, which makes possible to compute $\widehat{\Phi}^{t} \widehat{K} \widehat{\Phi}$ as $-\widehat{\Phi}^{t} C^{t} \Lambda$. In any case, when $\bar{K}_{r r}^{-1}$ stands for a preconditioned Krylov solver up to some tolerance, this approach leads to a generally nonsymmetric indefinite matrix. In these situations, it is better to use (11). Besides, we have used (11) in Section 6 due to slightly better performance.
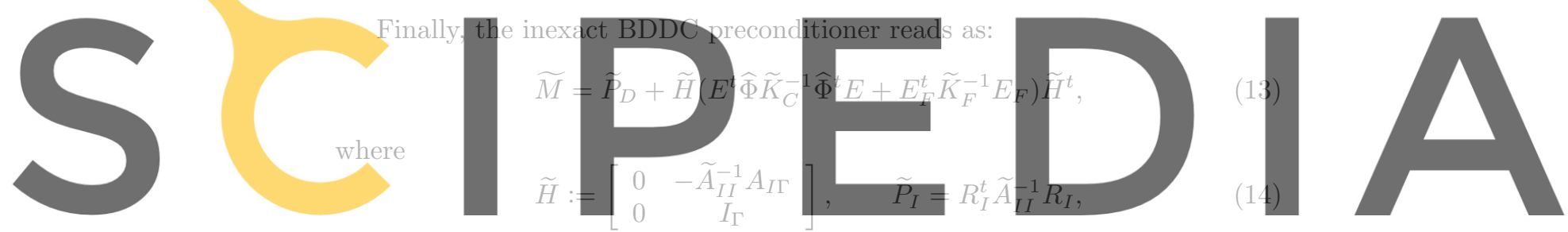

https//www.scipedia.com to download the version without the watermark

$$
\begin{aligned}
& \widehat{\Phi}^{(i)}=\left[\begin{array}{c}
\widehat{\Phi}_{c}^{(i)} \\
\widehat{\Phi}_{r}^{(i)}
\end{array}\right], \quad \widehat{\Phi}_{c}^{(i)}=\left[\begin{array}{ll}
I & 0
\end{array}\right], \quad \widehat{\Phi}_{r}^{(i)}=-\left(\widehat{K}_{r r}^{(i)}\right)^{-1} C_{r, i}^{t} \Lambda_{r}^{i}-\left[\begin{array}{ll}
K_{r c}^{(i)} & 0
\end{array}\right], \\
& C_{r, i}\left(\widehat{K}_{r r}^{(i)}\right)^{-1} C_{r, i}^{t} \Lambda_{r}^{(i)}=-C_{r, i}\left(\widehat{K}_{r r}^{(i)}\right)^{-1}\left[K_{r c}^{(i)} I\right] .
\end{aligned}
$$

Theorem 4.1. Let us assume that (8)-(10) hold. When $\widetilde{K}_{C}$ satisfies (11), we have:

$$
\lambda_{\min }(\widetilde{M} A) \geq \frac{\delta_{I} \min \left(1, \Delta_{F}^{-1}, \delta_{\Phi} \Delta_{C}^{-1}\right)}{\Delta_{\Phi} \Delta_{I}}, \quad \frac{\lambda_{\max }(\widetilde{M} A)}{\lambda_{\max }(M A)} \leq \frac{\Delta_{I}^{2} \max \left(1, \delta_{F}^{-1}, \Delta_{\Phi} \delta_{C}^{-1}\right)}{\delta_{\Phi} \delta_{I}^{2}} .
$$

Alternatively, when $\widetilde{K}_{C}$ satisfies (12), we get:

$$
\lambda_{\min }(\widetilde{M} A) \geq \frac{\delta_{I} \min \left(1, \Delta_{F}^{-1}, \Delta_{C}^{-1}\right)}{\Delta_{\Phi} \Delta_{I}}, \quad \frac{\lambda_{\max }(\widetilde{M} A)}{\lambda_{\max }(M A)} \leq \frac{\Delta_{I}^{2} \max \left(1, \delta_{F}^{-1}, \delta_{C}^{-1}\right)}{\delta_{\Phi} \delta_{I}^{2}} .
$$


Proof. The proof of this result readily follows from the analysis in [13], the only difference being the fact that the fine correction and $\Phi$ can in general be computed using different preconditioners. It can easily be handled using the fact that $K_{F}$ is a Galerkin projection of $K$ and a result like (11) for this matrix. Further, when the coarse preconditioner is built from $\widehat{\Phi}^{t} K \widehat{\Phi}$, the result is readily obtained using the fact that

$$
\frac{\delta_{C}}{\Delta_{\Phi}} x^{t} \widehat{\Phi}^{t} \widehat{K} \widehat{\Phi} x \leq x^{t} \widetilde{K}_{C} x \leq \frac{\Delta_{C}}{\delta_{\Phi}} x^{t} \widehat{\Phi}^{t} \widehat{K} \widehat{\Phi} x,
$$

which is obtained by combining (10)-(11).

Remark 4.2. In the inexact preconditioner $\widetilde{M}$ we have replaced the local/coarse problems by optimal approximations. Due to their block-diagonal structure, the (possibly different) preconditioners $\widetilde{K}_{r r}^{-1}$ and $\widehat{K}_{r r}^{-1}$ are locally built from $K_{r r}^{(i)}$, that can be obtained, e.g., as one $A M G$ cycle of this matrix. Analogously, $\widetilde{A}_{I I}^{-1}$ is built from local approximations of $A_{I I}^{(i)}$.

Remark 4.3. The preconditioners for the computation of $\Phi$ and the Dirichlet problems, i.e., $\widehat{K}_{r r}$ and $\widetilde{K}_{I I}$, must include the kernel correction (see [13]). On the other hand, it is not needed for the fine and coarse correction preconditioners $\widetilde{K}_{r r}$ and $\widetilde{K}_{C}$.

Remark 4.4. To compute $\widehat{\Phi}$, we must compute $\left(\widehat{K}_{r r}^{(i)}\right)^{-1} C_{r, i}^{t}$. It implies to apply at every subdomain the local preconditioner to as many vectors as local coarse corners. This computation can be reused to build the Schur complement matrix for the edge/face constrained Neumann problem in (4), as soon as $\widetilde{K}_{r r}=\widehat{K}_{r r}$. Otherwise, we must compute $\left(\widetilde{K}_{r r}^{(i)}\right)^{-1} C_{r, i}^{t}$. It makes suitable to consider the same preconditioner (with kernel correction) for both $\Phi$ and the fine correction.

\section{A highly scalable distributed-memory implementation}

In this section we adapt the highly scalable distributed-memory implementation of the method proposed in [12] to consider inexact solvers. The global linear system (1) is solved by means of a Krylov subspace method, where the inexact BDDC preconditioner $\widetilde{M}$ is used as a global system matrix preconditioner (see Section 4). In our implementation we can consider different Krylov subspace methods (e.g. PCG, IPCG [24], FGMRES [25]) which can be used for the solution of the global problem with a BDDC preconditioner or for the solution of the local problem with an AMG preconditioner [26, 3, 27]. This feature was exploited to test some combinations not reported here, e.g., PCGAMG methods for local problems (with coarse tolerances), and IPCG [24] for the global system. However, inexact variants based on Krylov methods turned out to be less efficient than a fixed number of $A M G$ cycles in all cases. These results have not been reported for the sake of brevity. 
In a distributed-memory implementation of a BDDC preconditioned Krylov subspace solver, all data structures (i.e., matrices and vectors) and computations are split and distributed among MPI tasks in concordance with the underlying non-overlapping partition of the domain. We refer the reader to [21] for a comprehensive coverage of these implementation aspects. In the rest of the section, we only identify and briefly describe those computations and communications required to implement the BDDC preconditioner with inexact solvers.

The initial set-up of the BDDC preconditioner is in turn split into a symbolic and a numerical phase in Algorithms 1 and 2, respectively, while its application to a residual is depicted in Algorithm 3. Communication stages are labeled as "GC" or "LC" depending on whether they are of global (i.e., all MPI tasks involved) or local (i.e., MPI tasks communicate with each other within subsets of tasks) nature, respectively. Algorithms 1, 2, and 3 require global gather/scatter communication, and local exchanges among nearest neighbors.

During the symbolic set-up of the BDDC preconditioner presented in Algorithm 1 , the adjacency graph (denoted by $G_{*}$ ) of matrices $A_{I I}^{(i)}$ and $K_{r r}^{(i)}$ required by the Dirichlet and constrained Neumann problems is computed in lines 12 and 13. The coarse solver tasks in lines 1- 11 are identical as for the exact BDDC method in [12].

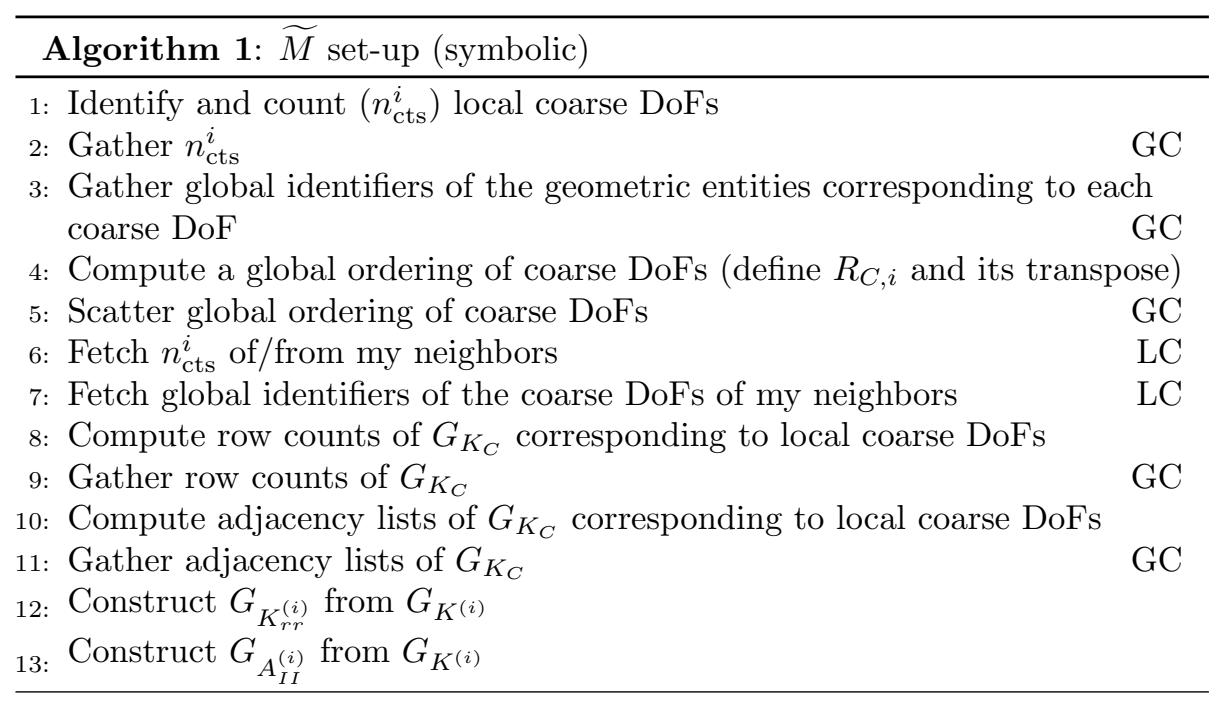

The numerical set-up of the BDDC preconditioner is presented in Algorithm 2. The operations required during this phase depend on the inexact solvers being used. E.g., the solver set-up is an incomplete numerical factorization for ILU methods, whereas it involves the construction of the hierarchy in AMG. The tasks in Algorithm 2 can be subdivided into fine tasks (lines 1-6) and coarse tasks (lines 7-9). Fine MPI tasks include the extraction of $A_{I I}^{(i)}$ and $K_{r r}^{(i)}$ in lines 1 and 2 and the set-up of their approximations, e.g., their AMG hierarchy and (possibly) the kernel-correction set-up, in lines 3 and 4, respec- 
tively. The fine duties also involve the computation of the coarse space matrix $\Phi$ in line 5 and the coarse matrix coefficients in line 6 . The MPI task (or tasks) in charge of the coarse problem then gathers these contributions and performs the matrix assembly corresponding to $R_{C, i}$ in order to build $K_{C}$ in lines 7 and 8, respectively. Finally, the MPI task in charge of the coarse problem performs the coarse preconditioner, e.g., the AMG hierarchy set-up of the inexact coarse matrix (see line 9).

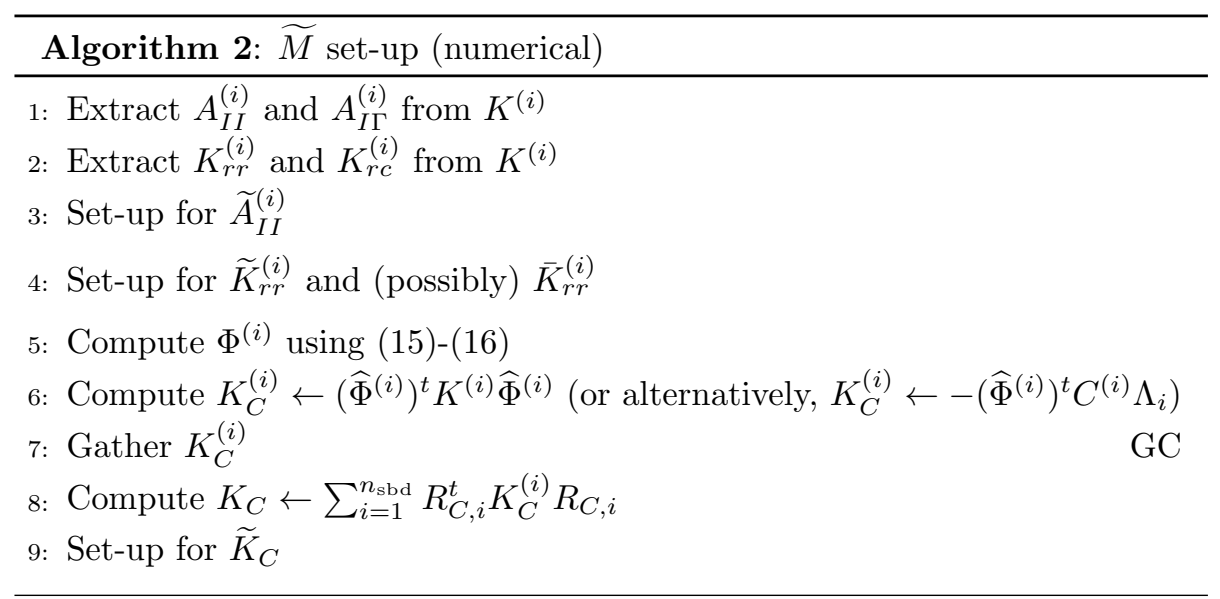

Algorithm 3 describes the algorithm that applies the BDDC preconditioner to a residual. First, the residual is extended to the BDDC space via $E^{t}$ (see line 1). On the one hand, the fine-grid tasks include the computation of the fine correction by means of constrained local Neumann problems (see line 4). Once the contributions from each subdomain to the coarse-grid residual are computed in line 2, the MPI tasks in charge of the coarse problem gather these contributions and perform the vector assembly associated to $R_{C, i}$ in order to build $r_{C}$ in lines 3 and 5, respectively. Next, the coarse problem is solved in an inexact way, e.g., by one/several AMG cycle(s). Finally, the solution is scattered from this task to all subdomains, so that all subdomains get the coarse-grid correction on its local coarse DoFs. Finally, both corrections are injected into $\overline{\mathbb{V}}$ via the projection $E$, and corrected in the interior in line 10.

The typical implementation of the BDDC preconditioner [28, 29] is illustrated in Figure 1 (a), where a one-to-one mapping between subdomains, MPI tasks, and computational cores is used. Fine and coarse duties are serialized. The vast majority of cores only have fine duties, and only some cores have both fine and coarse duties. This is due to the dramatic reduction of size between the original and coarse matrix. As a consequence, there is a tremendous amount of parallel overhead caused by idling, i.e., the wall-clock time required to solve the coarse problem $T_{C}$ times the number of cores with fine duties only. Further, cores with both coarse and fine duties require more memory resources. This is a problem for current multicore-based distributed-memory architectures (in the 


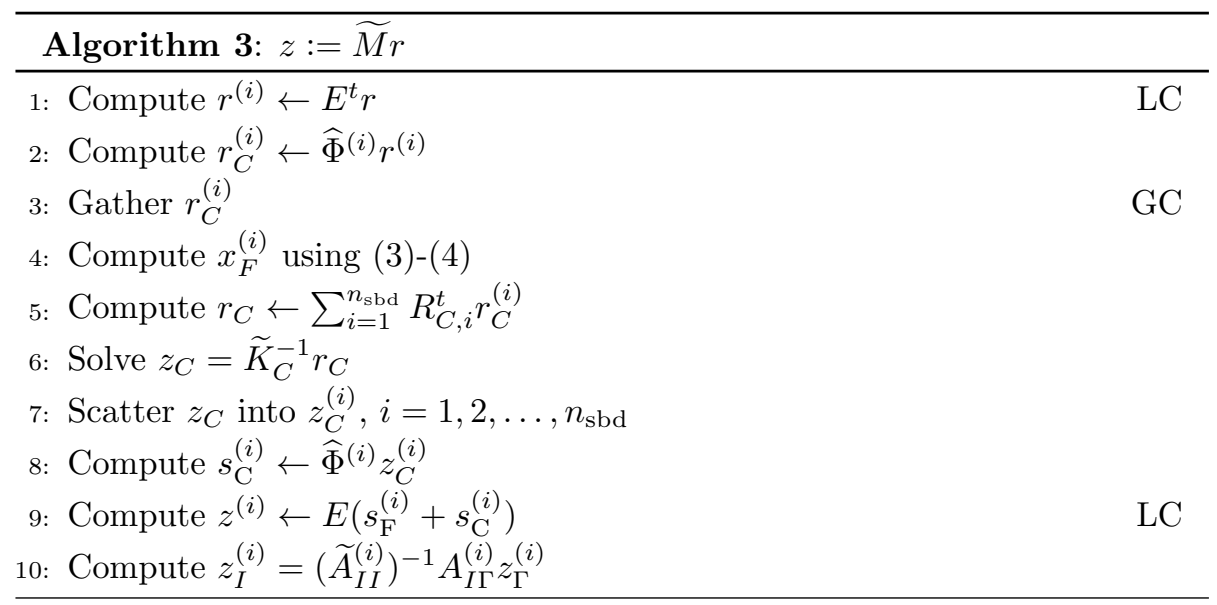

range 1-4 GBytes per core); these memory limitations are expected to be more restrictive in the future exascale supercomputers [1].

As an alternative, we have proposed in [12] a highly scalable implementation of the exact BDDC method that solves the aforementioned problems by exploiting the algorithmic property that makes possible to compute coarse and fine duties in parallel. This technique is illustrated in Figure 1 (b). The global set of MPI tasks (i.e., the global MPI communicator) is split into fine and coarse MPI tasks, i.e., those that have fine duties only (fine MPI communicator), and those with coarse duties only (coarse MPI communicator), so that the computation of fine and coarse corrections can be overlapped in time. Two possible approaches for the parallel solution of the coarse-grid problem are proposed in [12]: using an OpenMP coarse-grid solver within a dedicated node, as shown in Figure 1 (b), and its generalization into a MPI-based solution that distributes the coarse-grid problem.

The efficient exploitation of this idea requires an important remapping and re-scheduling of the communications and computations as well as some code refactoring, which is comprehensively described in [12]. The final result is depicted in Table 1, which is similar to the one in [12] but includes the modifications needed to use inexact solvers. Table 1 clearly evidences two areas or regions (three in the exact version [12], due to symbolic factorization), separated by global communication stages, where overlapping among fine and coarse duties is possible: the first one after gathering $K_{C}^{(i)}$ in line 7 of Algorithm 2 and the last one after gathering $r_{C}^{(i)}$ in line 3 of Algorithm 3. We stress the fact that all coarse duties, that produce severe idling and, as a result, a loss of parallel efficiency, can be overlapped with fine duties. Table 1 as a whole only considers the $\widetilde{M}$ set-up stages and the header of the Krylov phase. During the Krylov loop, overlapping among fine-grid/coarse-grid duties is present within each application of the preconditioner, as depicted on the region of Table 1 below the dashed horizontal line. 


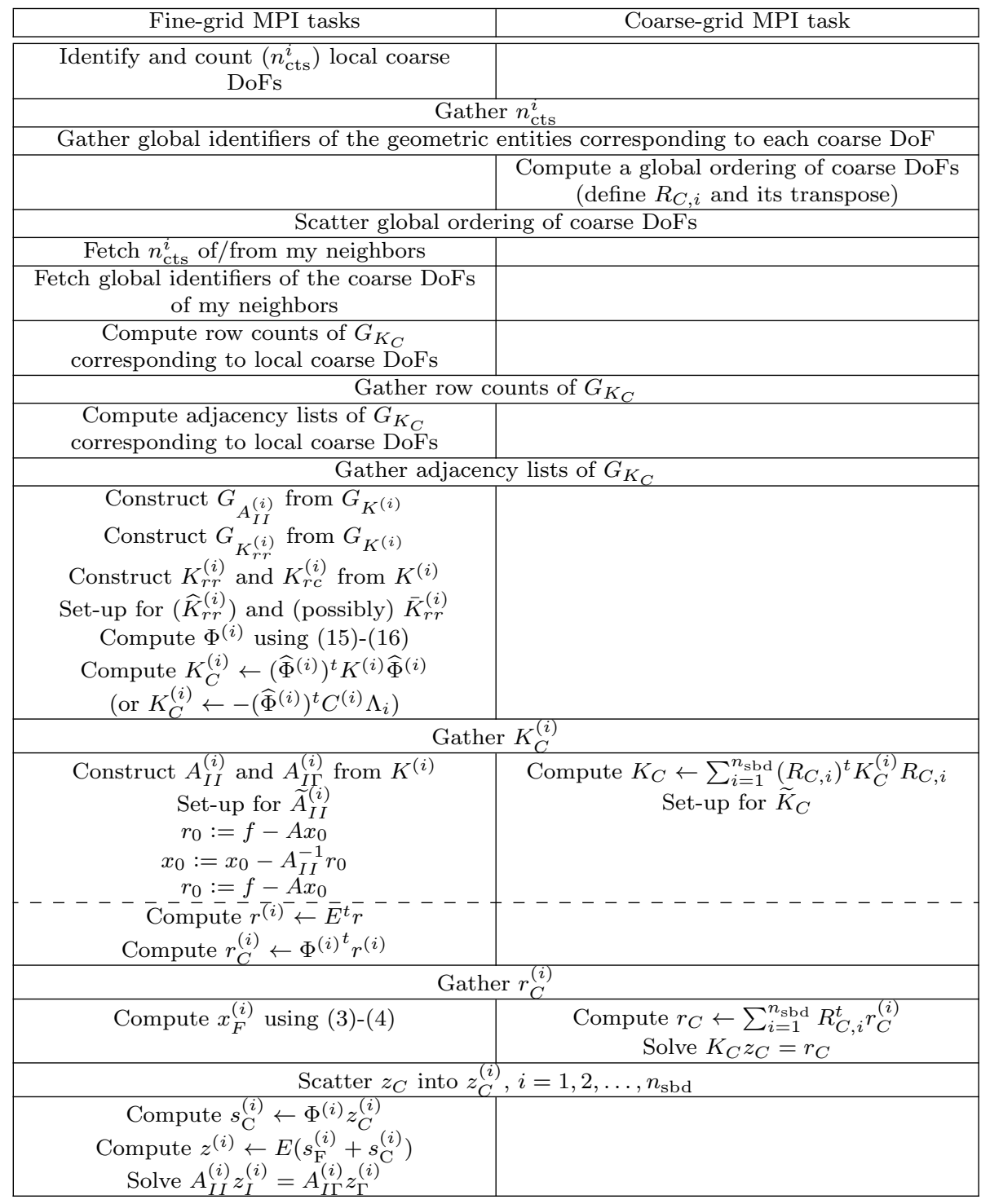

Table 1: Mapping of the PCG-BDDC algorithm to fine-grid and coarse-grid MPI tasks to achieve the maximum degree of overlapping in time. 


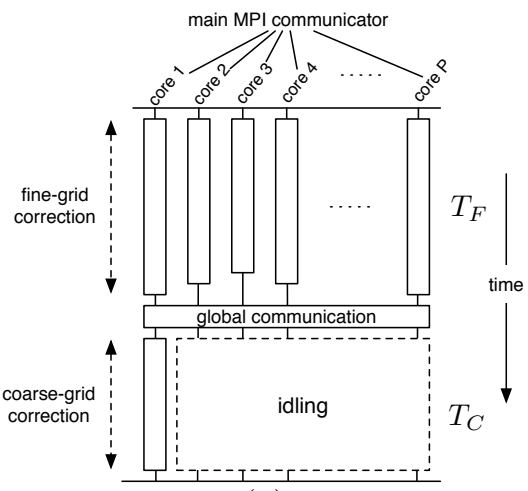

(a)

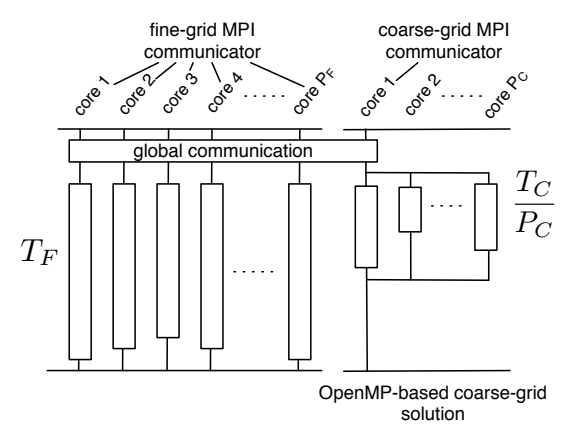

(b)

Figure 1: Comparison of (a) the typical parallel distributed-memory implementation of Algorithms 1, 2 and 3 and (b) the highly scalable one proposed in [12] implemented with multithreading.

\section{Numerical experiments}

The main goal underlying the numerical experiments section in this paper is to comprehensively assess, on a state-of-the-art supercomputer, the weak scalability of the overlapped implementation of the two-level BDDC preconditioner equipped with the machinery that allows to inexactly solve the internal problems (i.e., computation of coarse-grid space basis, local Dirichlet, and constrained Neumann problems, and global coarse-grid problem) while still preserving preconditioner optimality (see [13] and Section 4). The benefit of such techniques has to be viewed in the light of future parallel architectures: the trend is that that the most scalable architectures (e.g., IBM BlueGene) will have more limited memory per core. The study presented in the paper complements the mathematical analysis in [13] and answers how far can the overlapped/inexact BDDC codes go in the number of cores and the scale of the problem to still be within reasonable ranges of efficiency.

This section is structured as follows. Section 6.1 briefly introduces the parallel codes, and the software/hardware stack of the supercomputer on which they are tested. Section 6.2 describes the target problem, and the mapping of the parallel codes to the underlying supercomputer. Prior to the actual raw weak scalability study, in Section 6.3, we evaluate the effect that the inexact solution of each internal problem in isolation has on preconditioning efficiency (i.e., number of PCG iterations). In view of the results of this evaluation, in Section 6.4, we define a set of inexact BDDC variants that differ in the particular solvers used for each internal problem, leading to different trade-offs among total computation time versus preconditioner efficiency. Then, the weak scalability of these variants is comprehensively studied in order to meet the objectives of the section. 


\subsection{Code and parallel framework}

The inexact/overlapped implementation of the BDDC preconditioner to be studied in this paper has been implemented in the FEMPAR (Finite Element Multiphysics and massively PARallel) numerical software. FEMPAR is an inhouse developed, parallel hybrid OpenMP/MPI, object-oriented (OO) framework which, among other features, provides the basic tools for the efficient parallel distributed-memory implementation of substructuring DD solvers [21]. The parallel codes in FEMPAR heavily use standard computational kernels provided by (highly-efficient vendor implementations of) the BLAS and LAPACK. Besides, through proper interfaces to several third party libraries, the local fine-grid and the global coarse-grid problems in two-level DD methods can be solved by either sparse direct or approximate solvers. In this work, we explore HSL_MI20 [30] software package for the approximate solution of these problems. HSL_MI20 is a serial implementation of the classical Ruge-Stüben AMG preconditioner (as described, e.g., in [31] and [32]) to be used as a convergence accelerator of Krylov subspace solvers. AMG preconditioners, while being less robust than sparse direct methods in general, are particularly well-suited for systems arising from the discretization of the Poisson problem. Indeed, AMG preconditioning leads to optimal convergence rates (i.e., independent of mesh characteristic size) with linear arithmetic/memory complexity for a number of applications.

All experiments reported in this section were obtained on JUQUEEN, located in Jülich (Germany) at the Jülich Supercomputing Center (JSC). It belongs to the next generation of IBM Blue Gene family of supercomputers, the so-called BG/Q supercomputer. JUQUEEN is configured as a 28-rack system, featuring a total of 28,672 compute nodes interconnected by an extremely lowlatency five-dimensional (5D) torus interconnection network. Each compute node is equipped with a 16-core, 64-way threaded, IBM Power PC A2 processor, and 16 GBytes of SDRAM-DDR3 memory (i.e., 1GByte/core), and runs a lightweight proprietary CNK Linux kernel. The codes were compiled using IBM XLF Fortran compilers for BG/Q (v14.1) with recommended optimization flags. The customized MPICH2 library available on the system was used for message-passing. The codes were linked against the BLAS/LAPACK available on the single-threaded IBM ESSL library for BG/Q (v5.1), HSL_MI20 (v1.5.1), and HSL_MA87 (v2.1.1). At this point, it is worth noting that an effort was done to set up the HSL_MI20 parameters to reach the fastest solution times. In particular, the same subset of values for these parameters as those considered in [30] were tested, with $\theta=0.67$, RS1 coarsening, and Damped Jacobi smoothing being the winner combination for all internal problems. Besides, with this parameter-value combination, mesh independent convergence rates were achieved.

\subsection{Problem and parallel set-up}

We consider as benchmark the solution of the Poisson problem on a rectangular prism $\bar{\Omega}=[0,2] \times[0,1] \times[0,1]$ with homogeneous Dirichlet boundary 
conditions and a constant force term on the whole domain. A global conforming uniform mesh (partition) of $\bar{\Omega}$ into hexahedra is used for the trilinear FE discretization (i.e., Q1 FEs) of the continuous equation. The 3D mesh is partitioned into cubic grids of $P=4 m \times 2 m \times 2 m$ cubic subdomains. These subdomains are handled by as many MPI tasks as subdomains, which are distributed over $m^{3}=2^{3}, 3^{3}, \ldots, 18^{3}$ compute nodes $(128,432, \ldots, 93,312$ cores $)$, with $4 \times 2 \times 2$ subdomains/MPI tasks per compute node and one MPI task per physical core. An additional specialized MPI task is spawn in order to perform coarse-grid related computations. This task is mapped to an additional compute node, although it has only access to one core and 1 GByte of memory. This is due to limitations in the hardware/software stack of JUQUEEN, which does not allow to mix different execution modes on different compute nodes (e.g., 16 MPI tasks/ 1 thread per task on fine-grid nodes and 1 MPI task/16 threads per task on the coarse-grid node). Despite this, at first glance, severe restriction, we will demonstrate that these resources are already sufficient to solve very large-scale problems. ${ }^{1}$

The quotient among subdomain and mesh characteristic sizes, i.e., $\frac{H}{h}$, provides a measure of the local problem size. The number of FEs (i.e., hexahedra) on each local cubic subdomain is indeed $\frac{H}{h} \times \frac{H}{h} \times \frac{H}{h}$, and that of the global mesh is given by $4 m \frac{H}{h} \times 2 m \frac{H}{h} \times 2 m \frac{H}{h}$. The experiments performed in this section are selected in order to evaluate at which rate the computation time evolves with fixed $\frac{H}{h}$ and increasing number of cores (within the aforementioned range). As the trade-off among the factors determining the scalability of the codes depends on $\frac{H}{h}$, we perform the study with a pair of values of fixed problem size $\frac{H}{h}=40$ and 60 .

\subsection{The impact of approximately solving the internal problems}

In this section we evaluate the effect that the inexact solution of each internal problem in isolation has on the efficiency of the BDDC preconditioner. The objective of this section is two-fold. First, to confirm experimentally the results of the mathematical analysis presented in [13] and Section 4. Special attention will be paid on whether preconditioner optimality is preserved (i.e., number of PCG iterations asymptotically constant for fixed local problem size and increasing number of subdomains) no matter which of the internal problems is perturbed. Second, to determine to what extent there is margin for improvement (in terms of number of PCG iterations) by the usage of more accurate solvers for the internal problems. In such cases, it might be possible in practical scenarios to reach a trade-off among total computation time and preconditioner efficiency which leads to a faster solution of the problem.

Figures 2,3, and 4 compare the number of PCG iterations of the exact BDDC preconditioner with that of its inexact variant, for $\mathrm{BDDC}(\mathrm{c}), \mathrm{BDDC}(\mathrm{ce})$ and

\footnotetext{
${ }^{1}$ One way to deal with this restriction would be to distribute the coarse-grid problem among several MPI coarse-grid tasks, possibly spanning multiple compute nodes. This is not explored here, but left as future work.
} 
BDDC(cef), respectively. Each figure provides the impact that the perturbation of each internal problem in isolation has on the number of PCG iterations. For example, Figures 2 (a), 3 (a), and 4 (a) are focused on the impact of the inexact solution of the coarse-grid problem, while the rest of internal problems are solved exactly. The same applies for (b), (c), and (d), but for the solution of the Dirichlet problem, computation of coarse-grid basis, and solution of the constrained Neumann problem, respectively. For each experiment, three different inexact solvers were considered, namely "AMG(1)", "AMG(2)", and "AMG(4)", which stand for a single, two and four AMG cycles, respectively. The more AMG cycles, the more accurate the solution of the corresponding internal problem is expected to be, resulting in a beneficial impact on the number of inexact BDDC-PCG iterations. In Figures 2, 3, and 4, the global problem size was scaled linearly with the number of subdomains to keep a local problem size of $H / h=40$, i.e., 64K FEs per core; this is the largest local problem size that can be solved provided that the exact BDDC preconditioner implementation is based on sparse direct solvers, and the 1GByte/core memory constrain on JUQUEEN. The results obtained with smaller local problem sizes $(H / h=10,20,30)$ are omitted for brevity; similar conclusions to the ones with $H / h=40$ can be raised.

Figures 2, 3, and 4 overall confirm the mathematical analysis in [13] and Section 4. In particular, provided that the inexact BDDC preconditioner is equipped with spectrally equivalent approximations of the Neumann and coarse problems, and a spectrally equivalent kernel preserving approximations of the Dirichlet problem and the constrained Neumann problems at the computation of the coarse basis functions, preconditioner optimality is preserved. This can be observed in Figures 2, 3, and 4 by the number of PCG iterations being asymptotically constant no matter which of the internal problems is perturbed, and to what extent it is perturbed. While this is true, it is also worth noting that the impact that the inexact solution of the internal problems has on the number of PCG iterations is highly depending on the constraints considered for the coarse space, and the internal problem being perturbed. To see this, the $y$-axis of the four plots in Figures 2, 3, and 4 were scaled accordingly to the one corresponding to the internal problem whose perturbation has the largest impact on the number of PCG iterations.

If we focus on Figures 2 (a) and (c), it can be observed that the inexact solution/computation of the coarse-grid problem/coarse-grid basis has a very mild impact on the number of PCG iterations. Indeed, the number of PCG iterations of the inexact $\mathrm{BDDC}(\mathrm{c})$ preconditioner with a single $\mathrm{AMG}$ cycle is very close to that of the exact BDDC(c). Increasing the number of AMG cycles leads, as expected, to a reduction of the number of PCG iterations, which is almost negligible in this case. We consider this a very nice property of the BDDC preconditioning approach provided that the coarse-grid problem is the main scalability bottleneck, so that the overall solution approach immediately benefits from any savings in memory/time that can be achieved in this part of the algorithm. However, Figures 2 (b) and (d), reveal a high impact of the inexact solution of the Dirichlet and Neumann problems, respectively, with a 


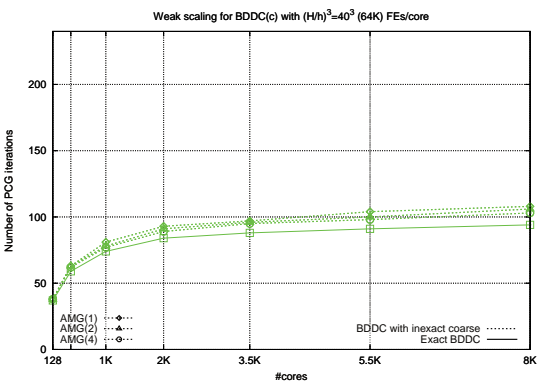

(a)

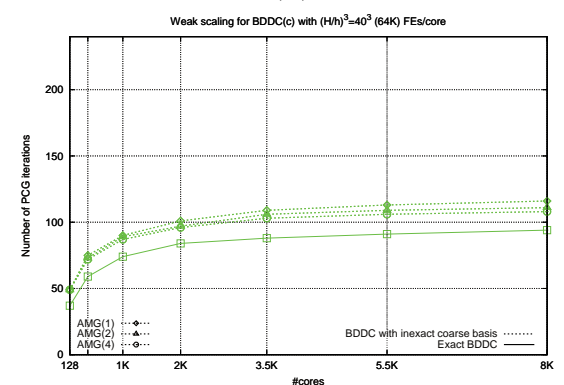

(c)

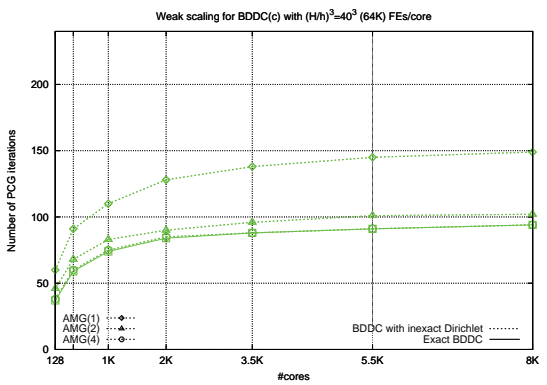

(b)

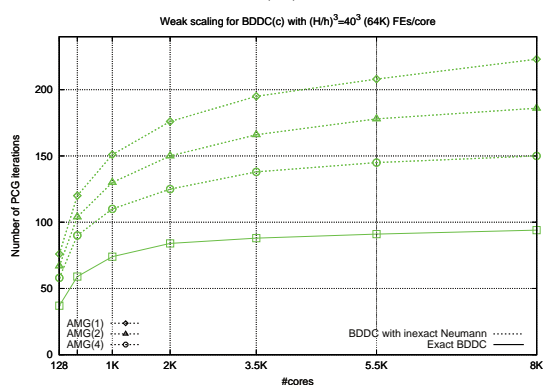

(d)

Figure 2: Sensitivity of the number of outer BDDC(c)-PCG iterations in the presence of perturbations in the solution of the (a) coarse, (b) Dirichlet, (c) coarse-grid basis, and (d) Neumann problems. Three different internal solvers, AMG(1), AMG(2) and AMG(4), were tested for the solution of these problems.

higher impact in the latter case. For example, with AMG(1), a roughly $50 \%$ and $100 \%$ increase in the number of PCG iterations with respect to the exact $\mathrm{BDDC}(\mathrm{c})$ preconditioner is observed, respectively. With additional AMG cycles for the approximation of the Dirichlet problem, the preconditioner efficiency of the exact $\mathrm{BDDC}(\mathrm{c})$ preconditioner can be rapidly recovered. However, in the case of the Neumann problem, still a $50 \%$ increase is observed with AMG(4).

The picture is quite different in the case of the $\mathrm{BDDC}(\mathrm{ce})$ preconditioner. While the inexact solution of the coarse-grid problem has a very mild impact on the number of PCG iterations (see Figure 3 (a)), the inexact computation of the coarse-grid basis has the highest impact (a roughly $80 \%$ increase in the number of PCG iterations for AMG(1)) among all internal problems. The impact of the inexact solution of the Dirichlet and Neumann problems is milder than that of the inexact computation of the coarse-grid basis, and similar to each other, with a $50 \%$ increase for AMG(1) (compare Figures 3 (b) and (c)).

Figures 4 (b) and (c) reveal a very close response of the BDDC(cef) preconditioner to that of the $\mathrm{BDDC}(\mathrm{ce})$ preconditioner in the presence of perturbations of the Dirichlet problem and computation of the coarse-grid basis, respectively. However, Figure 4 (a) reveals higher sensitivity of the BDDC(cef) precondi- 


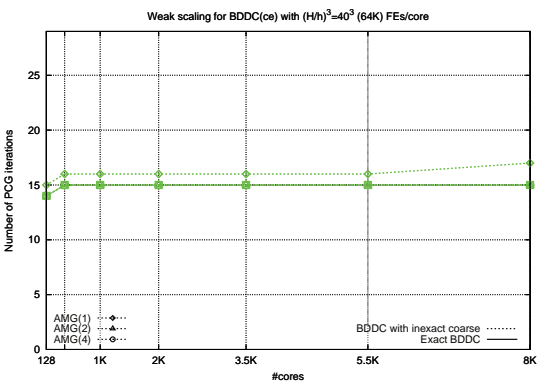

(a)

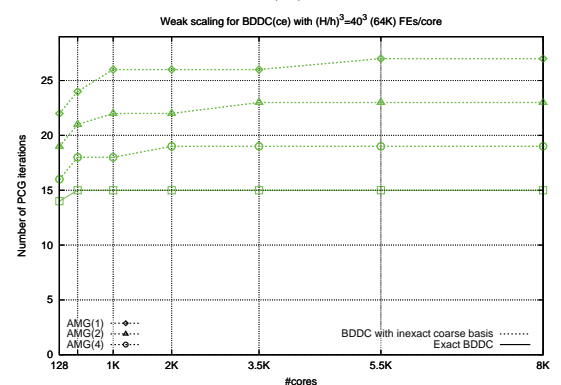

(c)

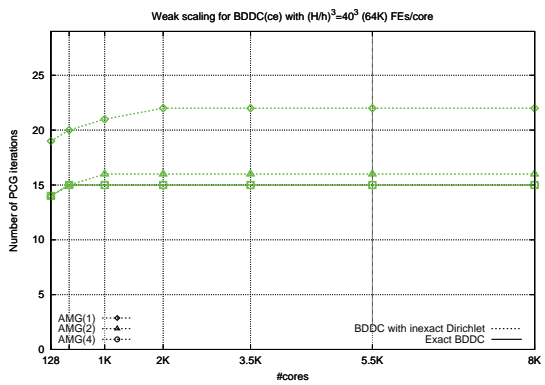

(b)

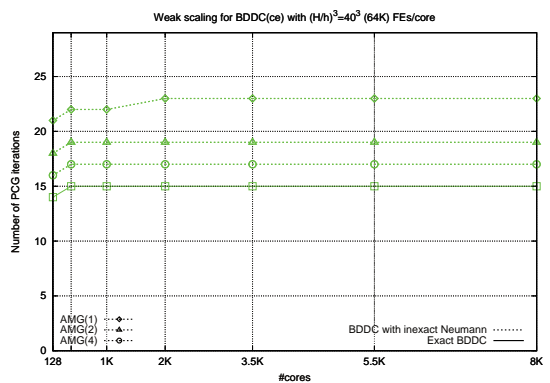

(d)

Figure 3: Sensitivity of the number of outer BDDC(ce)-PCG iterations in the presence of perturbations in the solution of the (a) coarse, (b) Dirichlet, (c) coarse-grid basis, and (d) Neumann problems. Three different internal solvers, AMG(1), AMG(2) and AMG(4), were tested for the solution of these problems.

tioner under perturbations in the solution of the coarse-grid problem. ${ }^{1}$ Indeed, with $\mathrm{AMG}(1)$, a $60 \%$ increase in the number of PCG iterations is observed with respect to the exact $\mathrm{BDDC}(\mathrm{cef})$ preconditioner. On the other hand, Figure 4 (d) reveals a very mild impact of the inexact solution of the Neumann problem, with AMG(1) already almost recovering the preconditioner efficiency of the exact $\mathrm{BDDC}(\mathrm{cef})$ preconditioner.

\subsection{Scalability of the overlapped implementation with inexact solvers}

In the previous section it has been shown that there is margin for improvement (at least in terms of the number of PCG iterations) by the usage of a more accurate solver than AMG(1) for the internal problems. In light of this observation, Table 2 presents a set of strategically selected inexact variants of the BDDC preconditioner. The columns labeled as " $\Phi "$, "Dirichlet", "Neumann", and "Coarse" refer to the computation of the coarse-grid basis vectors, Dirich-

\footnotetext{
${ }^{1}$ We remind that the $\mathrm{BDDC}(\mathrm{cef})$ coarse matrix is denser than the one for $\mathrm{BDDC}(\mathrm{ce})$. The AMG approximation seems to be less effective due to this fact.
} 


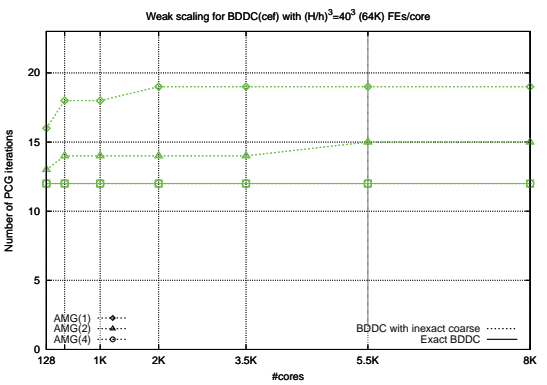

(a)

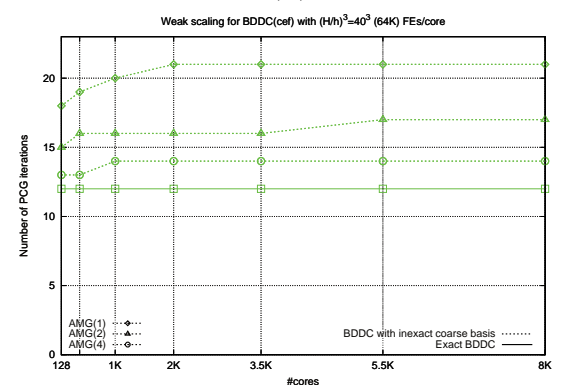

(c)

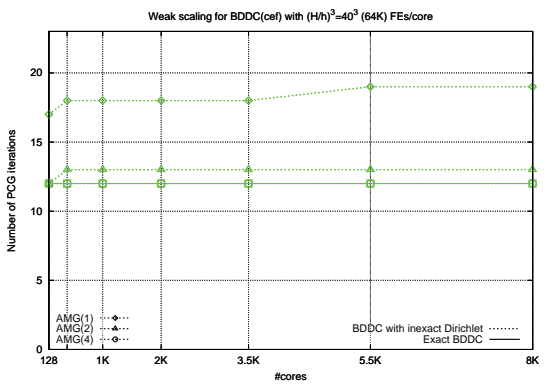

(b)

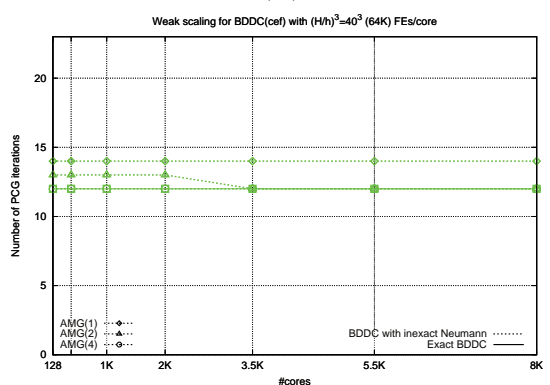

(d)

Figure 4: Sensitivity of the number of outer BDDC(cef)-PCG iterations in the presence of perturbations in the solution of the (a) coarse, (b) Dirichlet, (c) coarse-grid basis, and (d) Neumann problems. Three different internal solvers, AMG(1), AMG(2) and AMG(4), were tested for the solution of these problems.

let, Neumann, and coarse-grid internal problems, respectively. "AMG(1)", and "AMG(2)" stand for a single, and a pair of AMG cycles, respectively.

\begin{tabular}{ccccc} 
& $\Phi$ & Dirichlet & Neumann & Coarse \\
\hline Var. 1 & AMG(1) & AMG(1) & AMG(1) & AMG(1) \\
Var. 2 & AMG(1) & AMG(2) & AMG(1) & AMG(1) \\
Var. 3 & AMG(2) & AMG(1) & AMG(2) & AMG(1) \\
Var. 4 & AMG(2) & AMG(2) & AMG(2) & AMG(1) \\
\hline
\end{tabular}

Table 2: A set of strategically selected inexact variants of the two-level BDDC method .

We stress that the inner solver combinations that are shown in Table 2 are not the only ones possible, but the ones that have been strategically selected from a much wider set after comprehensive experimentation. First, we observed that it does not pay off a more accurate solver for the coarse-grid problem (e.g., AMG(2) or even an internal PCG-AMG iteration), as the reduction of the number of outer PCG iterations did not compensate for the decreased scalability at large core counts caused by a most costly solution of the coarse-grid problem. 
Second, we also considered variants where $\widetilde{K}_{r r} \neq \widehat{K}_{r r}$, e.g., $\operatorname{AMG}(2)$ for the Neumann problem, and AMG(1) for the computation of the coarse-grid vectors and vice versa. As stated in Remark 4.4, under this scenario one must compute $\left(\widetilde{K}_{r r}^{(i)}\right)^{-1} C_{r, i}^{t}$ to preserve symmetry, instead of re-using $\left(\widehat{K}_{r r}^{(i)}\right)^{-1} C_{r, i}^{t}$ from the constrained Neumann problem required for the computation of the coarse-grid basis vectors. This involves the solution of an extra linear system with multiple right-hand sides, as many as local coarse constraints, during preconditioner set-up. We experimentally observed that this extra computation significantly outweighs any gain derived from the usage of such variants.

A pair of details underlying the inexact variants in Table 2 are worth noting. First, the coarse-grid problem was built using the Galerkin projection of $K$ onto the inexact coarse basis $\widehat{\Phi}$ (see (11)) instead of the approach used in [13], that builds the coarse-grid problem as $\widehat{\Phi}^{t} \widehat{K} \widehat{\Phi}$ (see (12)). We consistently observed that the former approach leads at most to the same number of PCG iterations than the latter, with up to a $25 \%$ reduction in some cases (in particular, with the inexact BDDC(cef) preconditioner and the largest local problem size of $H / h=60)$. Second, although Var 3. and 4 put more effort than Var. 1 and 2, respectively, in the (more accurate) computation of the coarse-grid basis vectors and Neumann problem, note that in Var. 3 and 4 there is more potential for overlapping fine-grid and coarse-grid computations, in particular during preconditioner application at the bottom-most overlapping area of Table 1. We next study to what extent this property of Var. 3 and 4 leads to increased scalability and reduced computation times compared to those of Var. 1 and 2.

Figures 5 (a), (b), and (c) provide a comparative view of the weak scalability for the total computation time (in seconds) for the inexact variants of the $\mathrm{BDDC}(\mathrm{c}), \mathrm{BDDC}(\mathrm{ce})$ and $\mathrm{BDDC}(\mathrm{cef})$ solvers, respectively. The local problem sizes went from $40^{3}=64 \mathrm{~K}$ (left side) to $60^{3}=216 \mathrm{~K} \mathrm{FEs} \mathrm{per} \mathrm{core} \mathrm{(right} \mathrm{side),} \mathrm{while}$ the number of cores from 168 to 93,312 (see Section 6.2). For those variants, and local problem size combinations where a "high" degradation in the weak scalability was already observed up to $43.9 \mathrm{~K}$ cores, we did not run the codes beyond because we were limited in the consumption of the underlying parallel resources. On the other hand, Figures 6 (a), (b), and (c) report the number of PCG (outer solver) iterations for the same variants, and local problem size combinations in Figure 5. We set the initial solution vector guess $x_{0}=0$ for the outer iterations, that were stopped whenever the residual $r_{k}$ at a given iteration $k$ satisfies $\left\|r_{k}\right\|_{2} \leq 10^{-6}\left\|r_{0}\right\|_{2}$.

The shape of the different scalability curves shown in Figure 5 depends on the particular balance among fine-grid and coarse-grid computations achieved, for each inexact variant, in each of the three overlapping areas shown in Table 1, together with the preconditioner efficiency achieved by each variant, which determines the number of external outer solver iterations. For example, for the inexact BDDC(ce), and BDDC(cef) variants, and a load per core of $64 \mathrm{~K}$ FEs/core, the total computation time becomes dominated by the coarse-grid solver beyond $16 \mathrm{~K}$ and $8 \mathrm{~K}$ cores, respectively, rendering the overlapping technique less successful (i.e., given such load per core there is a limited potential for overlap- 

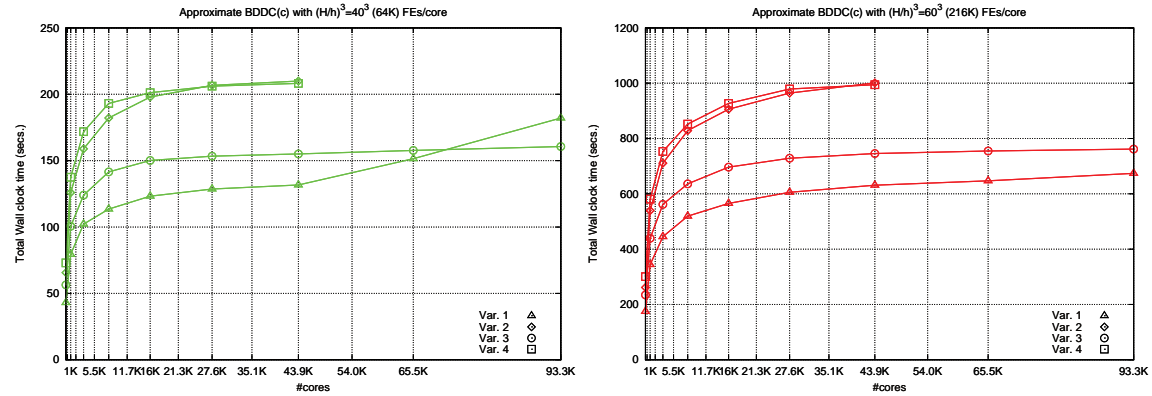

(a)
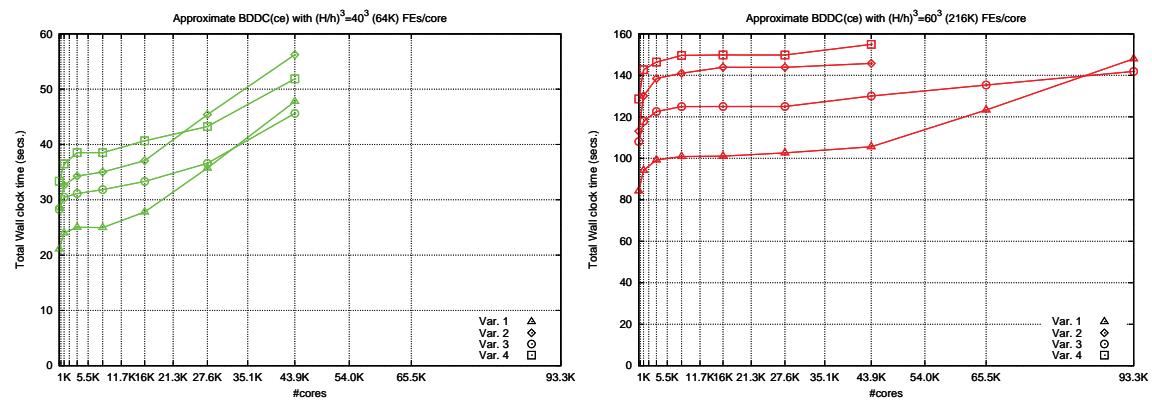

(b)
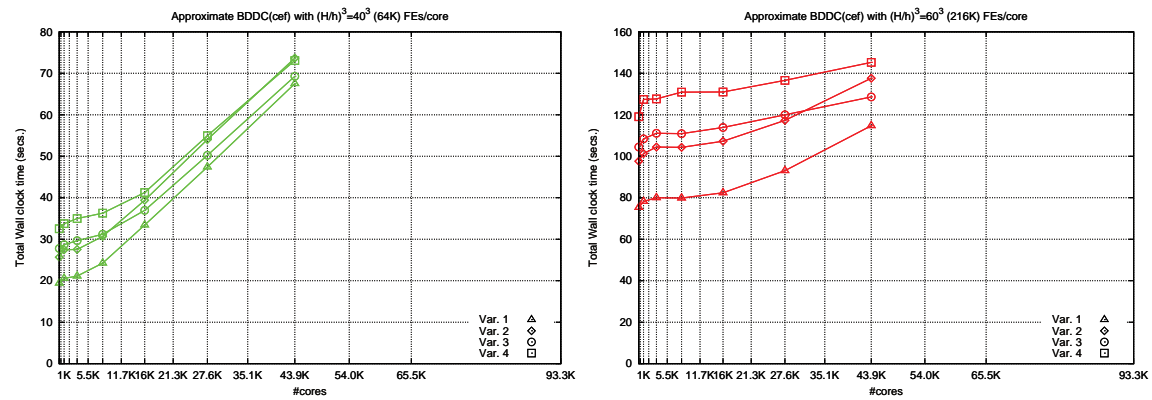

(c)

Figure 5: Weak scalability for the total computational time of the inexact variants of (a) BDDC(c), (b) BDDC(ce), and (c) BDDC(cef) solvers for the 3D Poisson problem on JUQUEEN. Left: $\frac{H}{h}=40$. Right: $\frac{H}{h}=60$. The solution of the coarse-grid linear system was mapped to an additional blade. 

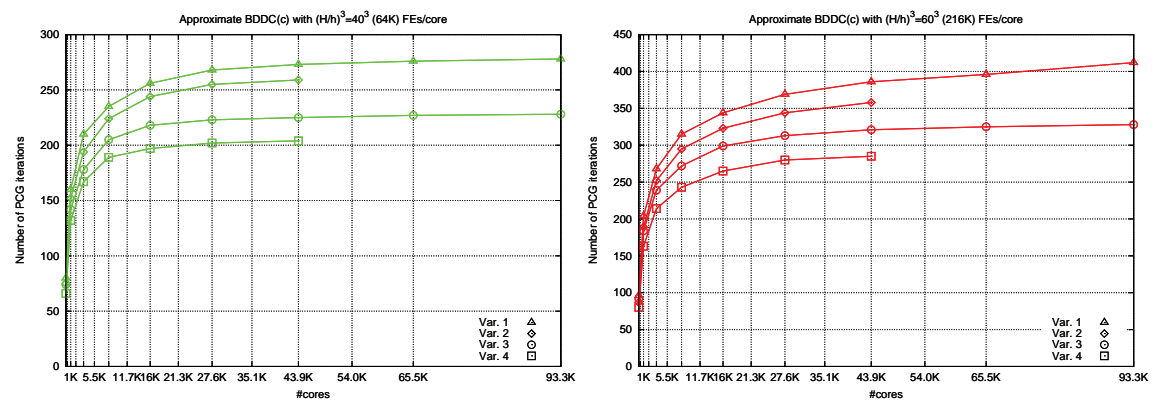

(a)
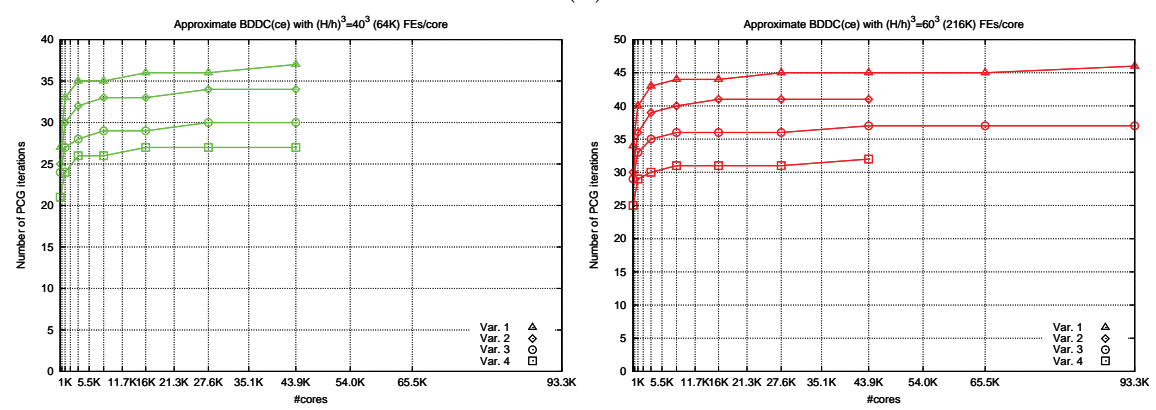

(b)
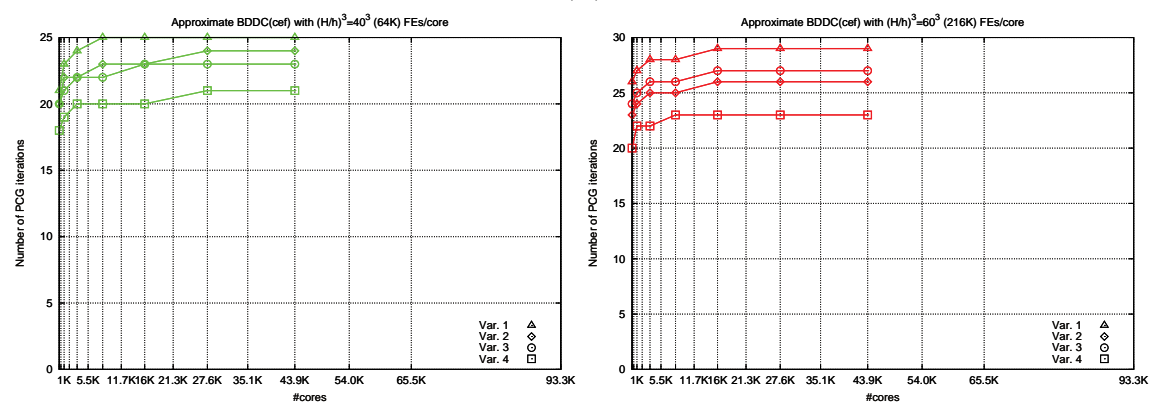

(c)

Figure 6: Weak scalability for the number of PCG iterations for the inexact variants of (a) $\operatorname{BDDC}(\mathrm{c})$, (b) $\operatorname{BDDC}(\mathrm{ce})$, and (c) BDDC(cef) solvers for the 3D Poisson problem on JUQUEEN. Left: $\frac{H}{h}=40$. Right: $\frac{H}{h}=60$. 
ping). This can be observed in the left-hand side of Figures 5 (b), and (c) by the total computation time of all variants for increasing number of cores. As expected, the degradation in the weak scalability is linear (with a higher slope in the case of inexact BDDC(cef) due to a larger, with denser stencil, coarse-grid problem) with the number of subdomains, given the linear arithmetic complexity of AMG preconditioning.

However, as the local problem size is increased, overlapping of fine-grid/coarsegrid duties becomes progressively more successful in tackling the bottleneck associated to the coarse-grid problem. For example, for inexact BDDC(c), Var. 3 , and a load per core of $64 \mathrm{~K}$ FEs per core, and for all variants in the case of the largest load per core of $216 \mathrm{~K} \mathrm{FEs/core,} \mathrm{the} \mathrm{weak} \mathrm{scalability} \mathrm{is} \mathrm{solely}$ determined by how fast the outer preconditioner solver achieves asymptotically constant converge rates with fixed problem size and increasing number of cores, meaning that the overlapping technique was successful to completely mask coarse-grid related computations in the 128-93.3K cores range; see the right-hand side of Figures 5 (a), and 6 (a). As shown in the right-hand side of Figure 5 (b), the same holds for inexact BDDC(ce), but in the $128-43.9 \mathrm{~K}$ cores range; a similar observation can be made for inexact BDDC(cef) with tighter core ranges in Figure 5 (c), due to a larger, with denser stencil, coarse-grid problem for the latter algorithm. Beyond $43.9 \mathrm{~K}$ cores, the computation time of coarse-grid problem related computations in overlapping areas \#1 and \#2 (see Table 1) starts exceeding that of the fine-grid related computations for the inexact $\mathrm{BDDC}(\mathrm{ce})$ variants, justifying the (very) mild degradation of roughly $20 \%$ and $50 \%$ that is observed for Var. 3 and 1, respectively, in the $43.9 \mathrm{~K}-93.3 \mathrm{~K}$ cores range, rendering a third level in the hierarchy necessary.

If we now turn our attention into Figures 6 (a), (b), and (c), we can observe that Var. 4 is consistently the one that leads to less number of iterations, followed by Var. 3, 2 and 1. This certainly makes sense given that Var. 4 is the one that solves more accurately all internal problems. However, in terms of computation times, and provided that the total computation times are dominated by fine-grid related computations, the relative rank of the variants subject of study change, with Vars. 1 and 3 being the faster, and Var. 4 the slowest; see Figures 5 (a), (b), and (c).

An interesting observation can be made, e.g., from the left hand side of Figure 6 (a) and Figure 6 (b), where Var. 3 becomes faster than Var. 1 for "sufficiently large" core counts, even with the extra computation time incurred by $\mathrm{AMG}(2)$ in the computation of the coarse-grid basis and the solution of the Neumann problem. As mentioned above, Var. 3 puts more computational effort in the solution of the Neumann problem. This increases the potential of the overlapped implementation to mask the solution of the coarse-problem at each preconditioner application during the PCG phase, resulting in increased scalability.

Further, it is very important to note the overall effect of a more accurate computation of the coarse system matrix on scalability. When the coarse basis is computed with AMG(2) (Vars. 3 and 4) instead of AMG(1) (Vars. 1 and 2), the scalability loss is much less severe. As expected, this degradation is linear, 
due to the linear complexity of AMG. However, the slope is noticeably worse for Vars. 1 and 2 than Vars. 3 and 4. As a conclusion, for large core counts, the reduced number of PCG iterations, increased scalability, and lower degradation make Var. 3 the winning choice at large core counts.

To end up with our study, we report in Table 3, for the exact and inexact variants of the BDDC preconditioner, and increasing values of the local problem size $\frac{H}{h}$, the memory consumption figures of the fine-grid preconditioning level; Table 4 reports those of the coarse-grid preconditioning level. ${ }^{1}$ The exact variant was supplied with HSL_MA87 [33], a highly-efficient parallel multi-threaded DAG-based code implementation of the supernodal sparse direct Cholesky solver. We note that the figures reported in Tables 3 and 4 correspond to the amount of (permanent) memory consumed by the preconditioner once it has been computed, and not to the (temporary) memory used during its computation.

As expected, the inexact variant of the BDDC preconditioner is less memory demanding than the exact one. This is clearly justified by the linear order of memory complexity of AMG solvers that the inexact variant fully exploits. In absolute terms, it consumes a moderate amount of memory, with roughly half a GByte for the largest local problem size. This is only a $50 \%$ of the memory available on JUQUEEN, meaning that larger problem sizes can still be solved on this machine (i.e., we did not still reach the memory limits of the proposed solver machinery), enabling improved scalability results. The (mild) increase of memory consumption with additional constraints can be easily explained by the fact that extra number of coarse-grid basis vectors have to be stored in memory. On the other hand, if we take a look at the memory consumption of the coarsegrid solver in Table 4 we observe, as expected, a moderate (linear) increase with the number of subdomains, with the higher the number of constraints, the higher the slope. In absolute terms, we can observe that for the largest number of subdomains tested (i.e., 93.3K), the inexact BDDC(ce) preconditioner consumed roughly a $58 \%$ (i.e., $582.7 \mathrm{MBytes}$ ) of the memory available, meaning that the solver machinery proposed can still solve larger problems on larger number of subdomains.

\section{Conclusions and future work}

In this work, we have analyzed the scalability of inexact BDDC preconditioners. Inexact AMG solvers are considered, due to their linear complexity and low memory requirements. Further, a highly scalable implementation of fine/coarse duties in time has been used, which is an extension of the work in [12] to inexact solvers. It allows us to mask the coarse problem tasks that harm scalability with embarrassingly parallel fine tasks and reduce both check-pointing

\footnotetext{
${ }^{1}$ Memory consumption was obtained on JUQUEEN by a call to malloc_stats right after preconditioner set-up.
} 


\begin{tabular}{ccrrrrr}
\hline & & \multicolumn{5}{c}{$\frac{H}{h}$} \\
\cline { 3 - 6 } Solver & Var. & 10 & 20 & 30 & 40 & 60 \\
\hline BDDC(c) & Inexact $20.8 \mathrm{M}$ & $38.2 \mathrm{M}$ & $81.2 \mathrm{M}$ & $157.1 \mathrm{M}$ & $516.5 \mathrm{M}$ \\
& Exact & $21.9 \mathrm{M}$ & $64.2 \mathrm{M}$ & $218.4 \mathrm{M}$ & $613.6 \mathrm{M}$ & O.M. \\
\hline BDDC(ce) & Inexact $22.6 \mathrm{M}$ & $42.3 \mathrm{M}$ & $86.7 \mathrm{M}$ & $158.9 \mathrm{M}$ & $522.2 \mathrm{M}$ \\
& Exact & $21.7 \mathrm{M}$ & $64.0 \mathrm{M}$ & $219.9 \mathrm{M}$ & $618.8 \mathrm{M}$ & O.M. \\
\hline BDDC(cef) & Inexact $22.6 \mathrm{M}$ & $42.3 \mathrm{M}$ & $86.7 \mathrm{M}$ & $160.6 \mathrm{M}$ & $527.5 \mathrm{M}$ \\
& Exact $21.7 \mathrm{M}$ & $64.3 \mathrm{M}$ & $219.6 \mathrm{M}$ & $625.2 \mathrm{M}$ & O.M. \\
\hline
\end{tabular}

Table 3: Memory consumption of the highest memory consuming fine-grid task for the exact and inexact variants of the 2-level BDDC preconditioner. O.M.: out of memory.

\begin{tabular}{ccrrrrrr}
\hline & \multicolumn{7}{c}{$m$ (\#subdomains $=16 \mathrm{~m}^{3}$ ) } \\
\cline { 2 - 8 } Solver & 6 & 8 & 10 & 12 & 14 & 16 & 18 \\
\hline Inexact BDDC(c) & $17.1 \mathrm{M}$ & $23.1 \mathrm{M}$ & $33.9 \mathrm{M}$ & $51.5 \mathrm{M}$ & $63.5 \mathrm{M}$ & $94.2 \mathrm{M}$ & $133.9 \mathrm{M}$ \\
\hline Inexact BDDC(ce) & $30.3 \mathrm{M}$ & $56.2 \mathrm{M}$ & $100.3 \mathrm{M}$ & $167.8 \mathrm{M}$ & $263.5 \mathrm{M}$ & $392.7 \mathrm{M}$ & $582.7 \mathrm{M}$ \\
\hline Inexact BDDC(cef) & $54.9 \mathrm{M}$ & $118.9 \mathrm{M}$ & $228.5 \mathrm{M}$ & $396.6 \mathrm{M}$ & $604.0 \mathrm{M}$ & O.M. & O.M. \\
\hline
\end{tabular}

Table 4: Memory consumption on coarse-grid task for the inexact variant of the 2-level BDDC preconditioner. O.M.: out of memory.

and idling. All these choices are motivated by the future exascale scenario, with very large core counts and reduced memory per core.

We have numerically tested the overlapped/inexact implementation of the algorithms in [13] (with a slight modification for the coarse solver approximation) that complements their mathematical analysis. This work shows how far the implementation proposed herein can scale with respect to the number of cores and the size of the global problem when using a serial AMG software package like HSL_MI20 [30].

As inexact solvers, we have considered a fixed (one or two) number of AMG cycles. (The use of PCG-AMG local/coarse solvers was considered, but it turned out to be less efficient than a fixed number of AMG cycles in all cases.) First, we have carried out a sensitivity analysis, to analyze the effect of inexact solvers on iteration counts/condition numbers. Next, we have performed a comprehensive weak scalability analysis till 93,312 cores and more than 20 billion unknowns on JUQUEEN, at the Jülich Supercomputing Center (JSC). As far as we know, these are the largest scale scalability analyses and simulations performed so far with DD methods. Even using a single core with 1 GByte of memory for the coarse-grid problem, the scalability of the inexact variants represent a dramatic improvement compared to the largest scale scalability analyses of exact BDDC methods so far (see [12]), justifying the approach considered herein.

Out of this analysis, we can conclude that for moderate core counts, less than $35 \mathrm{~K}$, the best option is to use one AMG cycle for all the local and coarse problems. However, as we run on larger sets of processors, to compute slightly more accurately the coarse basis and Neumann problems (using two AMG cycles) certainly pays the price; the resulting coarse problem is easier to approximate with AMG, the number of iterations is reduced, and there is more fine work 
load to mask the coarse tasks.

The next step in our effort to push forward balancing DD scalability till extreme core counts is to distribute the coarse-grid problem among several MPI coarse-grid tasks, possibly spanning multiple compute nodes. It can be accomplished by linking our inexact/overlapped BDDC implementation with a MPI-distributed AMG solver like BoomerAMG [17], or alternatively, to extend the overlapping BDDC techniques described above to a multilevel setting, using a recursive use of our implementation at FEMPAR. Based on our current experience (overlapped two-level implementations can scale up to several tens of thousands of processors) and existing mathematical analyses, we can naturally expect a three-level overlapped implementation of BDDC to perfectly scale in the largest HPC systems today. This is not explored here, but left as an exciting future line of research.

\section{Acknowledgements}

This work has been funded by the European Research Council under the FP7 Program Ideas through the Starting Grant No. 258443 - COMFUS: Computational Methods for Fusion Technology. A. F. Martín was also partially funded by the Generalitat de Catalunya under the program "Ajuts per a la incorporació, amb caràcter temporal, de personal investigador júnior a les universitats públiques del sistema universitari català PDJ 2013". We acknowledge PRACE for awarding us access to resource JUQUEEN based in Germany at JSC. We gratefully acknowledge JSC's staff in general, and Dirk Broemmel in particular, for their support in porting/debugging FEMPAR and its dependencies to/on JUQUEEN.

\section{References}

[1] Report on the workshop on extreme-scale solvers: Transition to future architectures, Tech. rep., U.S. Department of Energy (2012).

[2] P. T. Lin, J. N. Shadid, M. Sala, R. S. Tuminaro, G. L. Hennigan, R. J. Hoekstra, Performance of a parallel algebraic multilevel preconditioner for stabilized finite element semiconductor device modeling, Journal on Computational Physics 228 (17) (2009) 6250-6267. doi:http: //dx.doi.org/10.1016/j.jcp.2009.05.024.

[3] K. Stüben, A review of algebraic multigrid, Journal of Computational and Applied Mathematics 128 (12) (2001) 281 - 309. doi : 10.1016/S0377-0427(00)00516-1.

URL http://www.sciencedirect.com/science/article/pii/ S0377042700005161

[4] A. Toselli, O. Widlund, Domain Decomposition Methods - Algorithms and Theory, Springer-Verlag, 2005. 
[5] J. Mandel, Balancing domain decomposition, Communications in Numerical Methods in Engineering 9 (3) (1993) 233-241.

URL http://dx.doi.org/10.1002/cnm.1640090307

[6] C. R. Dohrmann, A preconditioner for substructuring based on constrained energy minimization, SIAM Journal on Scientific Computing 25 (1) (2003) 246-258. doi:10.1137/S1064827502412887. URL http://link.aip.org/link/?SCE/25/246/1

[7] C. Farhat, K. Pierson, M. Lesoinne, The second generation FETI methods and their application to the parallel solution of large-scale linear and geometrically non-linear structural analysis problems, Computer Methods in Applied Mechanics and Engineering 184 (2-4) (2000) 333-374. doi:10.1016/S0045-7825(99)00234-0.

URL http://www.sciencedirect.com/science/article/pii/ S0045782599002340

[8] S. Badia, A. F. Martín, J. Principe, Enhanced balancing NeumannNeumann preconditioning in computational fluid and solid mechanics, International Journal for Numerical Methods in Engineering 96 (4) (2013) 203-230.

[9] P. Amestoy, I. Duff, J.-Y. L'Excellent, Multifrontal parallel distributed symmetric and unsymmetric solvers, Computer Methods in Applied Mechanics and Engineering 184 (24) (2000) 501-520. doi: 10.1016/S0045-7825(99)00242-X.

URL http://www.sciencedirect.com/science/article/pii/ S004578259900242X

[10] B. Sousedík, J. Š́stek, J. Mandel, Adaptive-multilevel BDDC and its parallel implementation, ComputingIn press. doi: 10.1007/s00607-013-0293-5.

URL http://link.springer.com/article/10.1007/ s00607-013-0293-5

[11] V. Hapla, D. Horak, M. Merta, Use of direct solvers in TFETI massively parallel implementation, in: Applied Parallel and Scientific Computing, no. 7782 in Lecture Notes in Computer Science, Springer Berlin Heidelberg, 2013, pp. 192-205.

URL 978-3-642-36803-5_14

http://link.springer.com/chapter/10.1007/

[12] S. Badia, A. F. Martín, J. Principe, A highly scalable parallel implementation of balancing domain decomposition by constraints, SIAM Journal on Scientific Computing (2014) C190-C218doi:10.1137/130931989.

URL http://epubs.siam.org/doi/abs/10.1137/130931989

[13] C. R. Dohrmann, An approximate BDDC preconditioner, Numerical Linear Algebra with Applications 14 (2) (2007) 149168. doi:10.1002/nla. 514. 
URL

http://onlinelibrary.wiley.com/doi/10.1002/nla.514/

abstract

[14] X. Tu, Three-level BDDC in three dimensions, SIAM Journal on Scientific Computing 29 (4) (2007) 1759-1780. doi:10.1137/050629902.

URL http://epubs.siam.org/doi/abs/10.1137/050629902

[15] J. Mandel, B. Sousedík, C. Dohrmann, Multispace and multilevel BDDC, Computing 83 (2) (2008) 55-85. doi:10.1007/s00607-008-0014-7.

URL http://www.springerlink.com/content/112v4w1821r584u0/ abstract/

[16] J. Li, O. B. Widlund, On the use of inexact subdomain solvers for BDDC algorithms, Computer Methods in Applied Mechanics and Engineering 196 (8) (2007) 1415-1428. doi:10.1016/j.cma.2006.03.011.

URL http://www.sciencedirect.com/science/article/pii/ S0045782506002611

[17] V. E. Henson, U. M. Yang, BoomerAMG: a parallel algebraic multigrid solver and preconditioner, Applied Numerical Mathematics 41 (1) (2002) 155-177. doi:10.1016/S0168-9274(01)00115-5.

URL http://www.sciencedirect.com/science/article/pii/ S0168927401001155

[18] O. Rheinbach, Parallel iterative substructuring in structural mechanics, Archives of Computational Methods in Engineering 16 (4) (2009) 425-463. doi:10.1007/s11831-009-9035-4.

URL http://link.springer.com/article/10.1007/

s11831-009-9035-4

[19] A. Klawonn, O. Rheinbach, Highly scalable parallel domain decomposition methods with an application to biomechanics, ZAMM Journal of Applied Mathematics and Mechanics 90 (1) (2010) 532. doi:10.1002/zamm. 200900329 .

URL http://onlinelibrary.wiley.com/doi/10.1002/zamm. 200900329/abstract

[20] O. Schenk, K. Gärtner, On fast factorization pivoting methods for sparse symmetric indefinite systems, Electronic Transactions on Numerical Analysis 23 (2006) 158-179.

[21] S. Badia, A. F. Martín, J. Principe, Implementation and scalability analysis of balancing domain decomposition methods, Archives of Computational Methods in Engineering 20 (3) (2013) 239-262. doi:10.1007/ s11831-013-9086-4.

[22] J. Šístek, M. Čertíková, P. Burda, J. Novotný, Face-based selection of corners in 3D substructuring, Mathematics and Computers in Simulation 82 (10) (2012) 1799-1811. doi:10.1016/j.matcom.2011.06.007. 
URL http://www.sciencedirect.com/science/article/pii/ S0378475411001820

[23] J. Mandel, C. R. Dohrmann, Convergence of a balancing domain decomposition by constraints and energy minimization, Numerical Linear Algebra with Applications 10 (7) (2003) 639-659. doi:10.1002/nla.341.

URL http://dx.doi.org/10.1002/nla.341

[24] G. Golub, Q. Ye, Inexact preconditioned conjugate gradient method with inner-outer iteration, SIAM Journal on Scientific Computing 21 (4) (1999) 1305-1320. doi:10.1137/S1064827597323415. URL http://epubs.siam.org/doi/abs/10.1137/S1064827597323415

[25] Y. Saad, A flexible inner-outer preconditioned GMRES algorithm, SIAM Journal on Scientific Computing 14 (12) (1993) 461—469.

[26] P. Vaněk, J. Mandel, M. Brezina, Algebraic multigrid by smoothed aggregation for second and fourth order elliptic problems, Computing 56 (1996) 179-196.

URL http://dx.doi.org/10.1007/BF02238511

[27] M. Sala, R. Tuminaro, A new Petrov-Galerkin smoothed aggregation preconditioner for nonsymmetric linear systems, SIAM Journal on Scientific Computing 31 (1) (2008) 143-166. doi:10.1137/060659545.

[28] S. Balay, J. Brown, K. Buschelman, W. D. Gropp, D. Kaushik, M. G. Knepley, L. C. McInnes, B. F. Smith, H. Zhang, PETSc Web page, http: //www.mcs.anl.gov/petsc (2012).

[29] F. Hecht, FreeFem++ User's manual. 3rd edition, Version 3.22, available at http://www.freefem.org/ff++/ftp/freefem++doc.pdf (2013). URL http://www.freefem.org/ff++/ftp/freefem++doc.pdf

[30] J. Boyle, M. D. Mihajlović, J. A. Scott, HSL MI20: an efficient AMG preconditioner, Tech. Rep. RAL-TR-2007-021 (2007).

[31] J. W. Ruge, K. Stüben, Algebraic multigrid (AMG), in: Multigrid Methods, S. F. McCormick Edition, Vol. 3 of Frontiers in Applied Mathematics, SIAM, Philadelphia, PA, 1987, pp. 73-130.

[32] U. Trottenberg, C. C. W. Oosterlee, A. Schüller, MULTIGRID, Academic Press, 2001.

[33] J. Hogg, J. Reid, J. Scott, Design of a multicore sparse cholesky factorization using DAGs, SIAM Journal on Scientific Computing 32 (6) (2010) 3627-3649. doi:10.1137/090757216.

URL http://epubs.siam.org/doi/abs/10.1137/090757216 